\title{
Long non-coding RNA PAARH promotes hepatocellular carcinoma progression and angiogenesis via upregulating HOTTIP and activating HIF-1a/VEGF signaling
}

\author{
Huamei Wei $\mathbb{D}^{1,4}$, Zuoming $\mathrm{Xu}^{2,4}$, Liucui Chen ${ }^{3}$, Qing Wei ${ }^{3}$, Zihua Huang ${ }^{3}$, Guoman Liu ${ }^{3}$, Wenchuan Li ${ }^{2}$, Jianchu Wang ${ }^{2}$, \\ Qianli Tang (iD) ${ }^{2}$ and Jian Pu (iD) ${ }^{2 \times}$
}

(C) The Author(s) 2022

\begin{abstract}
Hepatocellular carcinoma (HCC) is one of the leading lethal malignancies and a hypervascular tumor. Although some long noncoding RNAs (IncRNAs) have been revealed to be involved in HCC. The contributions of IncRNAs to HCC progression and angiogenesis are still largely unknown. In this study, we identified a HCC-related IncRNA, CMB9-22P13.1, which was highly expressed and correlated with advanced stage, vascular invasion, and poor survival in HCC. We named this IncRNA Progression and Angiogenesis Associated RNA in HCC (PAARH). Gain- and loss-of function assays revealed that PAARH facilitated HCC cellular growth, migration, and invasion, repressed HCC cellular apoptosis, and promoted HCC tumor growth and angiogenesis in vivo. PAARH functioned as a competing endogenous RNA to upregulate HOTTIP via sponging miR-6760-5p, miR-6512-3p, miR-1298-5p, miR-6720-5p, miR-4516, and miR-6782-5p. The expression of PAARH was significantly positively associated with HOTTIP in HCC tissues. Functional rescue assays verified that HOTTIP was a critical mediator of the roles of PAARH in modulating HCC cellular growth, apoptosis, migration, and invasion. Furthermore, PAARH was found to physically bind hypoxia inducible factor-1 subunit alpha (HIF-1a), facilitate the recruitment of HIF-1a to VEGF promoter, and activate VEGF expression under hypoxia, which was responsible for the roles of PAARH in promoting angiogenesis. The expression of PAARH was positively associated with VEGF expression and microvessel density in HCC tissues. In conclusion, these findings demonstrated that PAARH promoted HCC progression and angiogenesis via upregulating HOTTIP and activating HIF-1a/VEGF signaling. PAARH represents a potential prognostic biomarker and therapeutic target for HCC.
\end{abstract}

Cell Death and Disease (2022)13:102; https://doi.org/10.1038/s41419-022-04505-5

\section{INTRODUCTION}

Hepatocellular carcinoma (HCC), the major primary liver cancer, is one of the most common malignancies and leading cause of cancer-related death [1]. Although great advances have been made in clinical interventions against $\mathrm{HCC}$, including surgical resection, hepatic transplantation, transcatheter arterial chemoembolization, and molecular targeted therapies, the prognosis of HCC is still very poor with 5 -year survival of $18 \%$ [2-5]. Thus, further revealing the molecular alterations involved in HCC initiation and progression would be beneficial for providing therapeutic targets for HCC $[6,7]$.

Except for the malignant phenotypes of HCC cells, tumor microenvironment (TME) also plays critical roles in promoting HCC development $[8,9]$. Hypoxia is one of the critical characteristics of most solid cancers, including HCC [10-13]. Hypoxia inducible factor-1a (HIF-1a) is stabilized by hypoxia [14]. HIF-1a activates the expression of its targeted genes, such as VEGF, which induces angiogenesis $[15,16]$. Angiogenesis is well-known to be involved in HCC $[17,18]$. HIF-1a/VEGF signaling axis-induced angiogenesis has also been revealed in HCC $[14,19]$. Anti-angiogenesis antibody ramucirumab has been approved by US FDA as a second line treatment for advanced HCC patients, supporting the crucial roles of angiogenesis in HCC. However, the molecular mechanisms underlying the angiogenesis of HCC are still largely unknown.

Increasing evidences in transcriptome sequencings have identified more and more non-coding transcripts [20-22]. Among these transcripts, long non-coding RNAs (IncRNAs) are a class of regulatory non-coding RNAs with more than 200 nucleotides in length [23-25]. IncRNAs have been revealed to play various roles in multiple pathophysiological processes, including HCC [26-32]. For instance, IncRNA-ATB promotes the invasion-metastasis cascade in HCC [33]. IncRNA GPC3-AS1 promotes HCC cellular proliferation and migration [34]. IncRNA ANCR promotes HCC metastasis [35]. The molecular mechanisms of IncRNAs are also complex and various [36,37]. One of the important mechanisms of cytoplasmic IncRNAs is to competitively bind microRNAs (miRNAs) $[38,39]$. miRNAs are another class of regulatory non-coding RNAs with about 19-25 nucleotides in length. miRNAs are well-known to

\footnotetext{
${ }^{1}$ Department of Pathology, Affiliated Hospital of Youjiang Medical University for Nationalities, Baise, China. ${ }^{2}$ Department of Hepatobiliary Surgery, Affiliated Hospital of Youjiang Medical University for Nationalities, Baise, China. ${ }^{3}$ Graduate College of Youjiang Medical University for Nationalities, Baise, China. ${ }^{4}$ These authors contributed equally: Huamei Wei, Zuoming Xu. ${ }^{凶}$ email: jian_pu@126.com

Edited by Dr. George Calin
}

Received: 20 August 2021 Revised: 8 December 2021 Accepted: 30 December 2021

Published online: 02 February 2022 
inhibit their target genes expression $[40,41]$. IncRNAs relieve the repressive roles of miRNAs on their targets. These IncRNAs were also classed as competing endogenous RNA (ceRNA). For instance, IncRNA-ATB upregulated ZEB1 and ZEB2 through competitively binding miR-200 family [33]. IncRNA HNF1A-AS1 upregulated PIK3CD via competitively binding miRNA-30b-3p [38]. Another mechanism of action of IncRNAs is to physiology bind proteins and modulate the expression and/or function of the interacted proteins. For instance, GPC3-AS1 physically binds PCAF, recruits PCAF to GPC3, and activates GPC3 expression [34]. MRCCAT1 physically binds PRC2, recruits PRC2 to NPR3, and represses NPR3 transcription [42]. Apart from the important roles of IncRNAs, aberrant expressions of IncRNAs are frequently revealed in a variety of diseases [43]. However, the potential contributions of IncRNAs to HCC TME, particular angiogenesis are still largely unclear.

In this study, we identified a novel HCC-related IncRNA CMB922P13.1 (gene ID: 101927789; accession number: NR_160549) via analyzing the cancer genome atlas (TCGA) liver hepatocellular carcinoma (LIHC) dataset. We further investigated the expression, roles, and mechanism of action of this IncRNA in HCC. Our findings revealed that this IncRNA not only enhanced malignant phenotypes of HCC cells, but also promoted angiogenesis. Therefore, we named this IncRNA Progression and Angiogenesis Associated RNA in HCC (PAARH).

\section{MATERIALS AND METHODS \\ Bioinformatics analyses}

PAARH expression in HCC and the correlations between PAARH expression and overall survival and disease-free survival of HCC patients based on the RNA sequencing data of TCGA LIHC project were analyzed using the online in silico tool Gene Expression Profiling Interactive Analysis (GEPIA) (http:// gepia.cancer-pku.cn/) [44]. Gene expression correlation in HCC tissues based on TCGA LIHC dataset was calculated using GEPIA (http://gepia. cancer-pku.cn/). Coding potential of PAARH was analyzed using Coding Potential Assessment Tool (CPAT) (http://lilab.research.bcm.edu/) [45], Coding Potential Calculator (CPC) 2.0 (http://cpc2.gao-lab.org/) [46], and TestCode (https://www.bioinformatics.org/sms/testcode.html). The miRNAs binding to PAARH and HOTTIP were predicted by LncBase Predicted v.2 (http://carolina.imis.athena-innovation.gr/diana_tools/web/index.php? $r=$ Incbasev2\%2Findex-predicted) [47]. The binding between PAARH and HIF-1a was predicted by RNA-Protein Interaction Prediction (RPISeq) (http://pridb.gdcb.iastate.edu/RPISeq/index.html) [48].

\section{Clinical samples}

Seventy-two pairs of HCC tissues and adjacent noncancerous liver tissues were acquired at the Affiliated Hospital of Youjiang Medical University for Nationalities from HCC patients who received surgical resection with written informed consents. All tissues were diagnosed by two experienced pathologists. The clinicopathological characteristics of these 72 HCC cases were shown in Supplementary Table 1. This study was undertaken following the Declaration of Helsinki and approved by the Ethics Committee of Affiliated Hospital of Youjiang Medical University for Nationalities.

\section{Cell cultures}

Human HCC cell lines SNU-398 and SK-HEP-1, and Human umbilical vein endothelial cell (HUVEC) were purchased from American Type Culture Collection (ATCC, Manassas, VA, USA). Human HCC cell line Huh7 was purchased from National Collection of Authenticated Cell Cultures of Chinese Academy of Sciences (Shanghai, China). SNU-398 was maintained in RPMI 1640 medium (Invitrogen, Carlsbad, CA USA) added with 10\% fetal bovine serum (FBS, Invitrogen). SK-HEP-1 was maintained in Eagle's Minimum Essential Medium (Invitrogen) added with 10\% FBS. HUVEC was maintained in Vascular Cell Basal Media (ATCC) supplemented with Endothelial Cell Growth Kit (ATCC). Huh7 was maintained in Dulbecco's modified Eagle's medium (Invitrogen) added with 10\% FBS. All cells were cultured at $37{ }^{\circ} \mathrm{C}$ containing $5 \% \mathrm{CO}_{2}$ under normoxia. Under hypoxia, cells were cultured at $37^{\circ} \mathrm{C}$ containing $1 \% \mathrm{O}_{2}, 94 \% \mathrm{~N}_{2}$, and $5 \% \mathrm{CO}_{2}$. The cells were authenticated using STR profiles. All cells were routinely tested as mycoplasma-free.

\section{Quantitative real-time polymerase chain reaction (qRT-PCR)}

Total RNA was isolated from indicated tissues and cells using TRIzol Reagent (Invitrogen). Next, the RNA was used to perform reverse transcription to generate complementary DNA (cDNA) by the M-MLV Reverse Transcriptase (Invitrogen) and random primers. The CDNA was further used to perform qRT-PCR by the TB Green Premix Ex Taq II (TaKaRa, Tokyo, Japan) on StepOnePlus Real-Time PCR System (Applied Biosystems, Foster City, CA, USA). The primer sequences were 5'-CCCTGAAGA TAAGGTTGTGC-3' (sense) and 5'-CTGTGGTTTGCTGAGGAGTT-3' (anti-sense) for PAARH， 5'-CCTCCTCACCAAAAATGG-3' (sense) and 5'-GGCAGGGCTG TACTCAAAT-3' (anti-sense) for HOTTIP, 5'-AGGAGTACCCTGATGAGAT-3' (sense) and $5^{\prime}$-GCCTTGGTGAGGTTTGAT-3' (anti-sense) for VEGF, 5'-TG GAGAAATAGTAGATGGC-3' (sense) and $5^{\prime}$-GGTGAGGAAGTAAAAACAG-3' (anti-sense) for MALAT1, 5'-GTCGGAGTCAACGGATTG-3' (sense) and 5'-TG GGTGGAATCATATTGGAA-3' (anti-sense) for GAPDH. GAPDH was used as an endogenous control. For the quantification of miRNAs, TaqMan Advanced miRNA Assays (Thermo Fisher, Waltham, MA, USA) were performed on StepOnePlus Real-Time PCR System following the manufacturer' protocols. Relative quantification was calculated using the comparative $\mathrm{Ct}$ method.

\section{Plasmids construction and transfection}

PAARH full-length sequences were PCR-amplified with the primers $5^{\prime}-\mathrm{C}$ CCAAGCTTGCACACTCTTGAAGTGACCG-3' (sense) and 5'-CGGGATCCGA GATGGAGTCTCGCTCTTG-3' (anti-sense). The PCR products were cloned into the Hind III and BamH I sites of pcDNA3.1 (+) (Invitrogen) to generate PAARH overexpression plasmid pcDNA3.1-PAARH. Furthermore, the PCR products were also cloned into the Hind III and BamH I sites of pSPT19 (Roche, Basel, Switzerland) to generate PAARH in vitro transcription plasmid pSPT19-PAARH. Two pairs of CDNA oligonucleotides targeting PAARH were synthesized and cloned into the shRNA lentivirus expressing plasmid pLV3/H1/GFP\&Puro (GenePharma, Shanghai, China). One pairs of CDNA oligonucleotides targeting HOTTIP were synthesized and cloned into pLV3/H1/GFP\&Puro. The constructed plasmid was co-transfected with pGag/Pol, pRev and pVSV-G (GenePharma) into HEK-293FT cells to generate shRNA lentivirus targeting PAARH or HOTTIP. Scrambled non-targeting shRNA lentivirus were used as negative control (NC). The shRNA oligonucleotide sequences were $5^{\prime}$ GATCCGCAAGTCAACCCTGTACAACCTTCAAGAGAGGTTGTACAGGGTTGACTTG CTITTTG-3' (sense) and 5'-AATTCAAAAAAGCAAGTCAACCCTGTACAACCTC TCTTGAAGGTTGTACAGGGTTGACTTGCG-3' (anti-sense) for shRNA-PAARH-1, 5'-GATCCGCCTGAAGACACAGGACAATGTTCAAGAGACATTGTCCTGTGTCTTCAG GCTITITTG-3' (sense) and 5'-AATTCAAAAAAGCCTGAAGACACAGGACAATG TCTCTTGAACATTGTCCTGTGTCTTCAGGCG-3' (anti-sense) for shRNA-PAARH-2, 5'-GATCCGTCCTGACCAATGTAAGTGTCTTCAAGAGAGACACTTACATTGGTCAGG ACTITाTG-3' (sense) and 5'-AATTCAAAAAAGTCCTGACCAATGTAAGTGT CTCTCTTGAAGACACTTACATTGGTCAGGACG-3' (anti-sense) for shRNA-HOTTIP, 5'-GATCCGTTCTCCGAACGTGTCACGTTTCAAGAGAACGTGACACGTTCGGAGAA CTITITTG-3' (sense) and 5'-AATTCAAAAAAGTTCTCCGAACGTGTCACGTTC TCTTGAAACGTGACACGTTCGGAGAACG-3' (anti-sense) for shRNA-NC. PAARH sequences containing miR-6760-5p, miR-6512-3p, miR-1298-5p, miR-6720-5p, miR-4516, and miR-6782-5p binding sites were PCR-amplified with the primers $5^{\prime}$-CGCTCGAGAAGTGACCGGCCTGGAGA-3' (sense) and 5'-GCTCTAG AGTGCAGTGGCACGATCTCG-3' (anti-sense). The PCR products were cloned into the Xho I and Xba I sites of pmirGLO Dual-Luciferase miRNA Target Expression Vector (Promega, Madison, WI, USA) to generate pmirGLO-PAARH. HOTTIP sequences containing miR-6760-5p, miR-6512-3p, miR-1298-5p, miR6720-5p, miR-4516, and miR-6782-5p binding sites were PCR-amplified with the primers $5^{\prime}$-CTAGCTAGCGTGAAAGTGGGCACATTA-3' (sense) and 5'-CC GCTCGAGAACAAAAGAAACCAGAACAT-3' (anti-sense), followed by being cloned into the Nhe I and Xho I sites of pmirGLO to generate pmirGLOHOTTIP. The MS2-12x fragment was PCR-amplified from pSL-MS2-12 $\times$ (Addgene, Watertown, MA, USA) with the primers $5^{\prime}$-ATGATATCCCGGGCCC TATATATGGATC- $3^{\prime}$ (sense) and $5^{\prime}$-CCGCTCGAGTATCGATCGCGCGCAGATCT A-3' (anti-sense) [49], followed by being cloned into the EcoR $V$ and Xho I sites of pcDNA3.1 or pcDNA3.1-PAARH to generate pcDNA3.1-MS2-12X or pCDNA3.1-PAARH-MS2-12x. VEGF promoter sequences were PCR-amplified with the primers $5^{\prime}$-GGGGTACCTAGCACCTCCACCAAACC-3' (sense) and $5^{\prime}$-CC CAAGCTTCACAGCCTGAAAATTACCC- $3^{\prime}$ (anti-sense), followed by being cloned into the Kpn I and Hind III sites of pGL3-Basic Luciferase Reporter Vector to generate $\mathrm{pGL} 3-V E G F$ promoter.

miRNAs, siRNAs, and transfection

miR-6760-5p, miR-6512-3p, miR-1298-5p, miR-6720-5p, miR-4516, and miR$6782-5 p$ mimics and NC were purchased from Thermo Fisher. ON- 
TARGETplus Human DICER1 siRNA SMARTpool was purchased from Dharmacon (Cambridge, England). The transfection and co-transfection of miRNAs, siRNAs, and plasmids were undertaken with Lipofectamine 3000 (Invitrogen).

\section{Stable cell lines construction}

To construct PAARH stably overexpressed and control HCC cells, PAARH overexpression plasmid pcDNA3.1-PAARH or empty plasmid pcDNA3.1 was transfected into SNU-398 and SK-HEP-1 cells. Forty-eight hours after transfection, the cells were treated with $800 \mu \mathrm{g} / \mathrm{ml}$ neomycin for four weeks to select PAARH overexpressed cells. To construct PAARH stably silenced and control HCC cells, SUN-398 and Huh7 cells were infected with shRNA lentivirus targeting PAARH or scrambled non-targeting shRNA lentivirus. Ninety-six hours after infection, the cells were treated with $2 \mu \mathrm{g} /$ $\mathrm{ml}$ puromycin for four weeks to select PAARH silenced cells. To construct PAARH overexpressed and concurrently HOTTIP depleted HCC cells, PAARH overexpressed SNU-398 cells were infected with shRNA lentivirus targeting HOTTIP. Ninety-six hours after infection, the cells were treated with $2 \mu \mathrm{g} / \mathrm{ml}$ puromycin and $800 \mu \mathrm{g} / \mathrm{ml}$ neomycin for four weeks to select PAARH overexpressed and concurrently PAARH silenced cells.

\section{Cell growth, apoptosis, migration, and invasion assays}

Cell growth was evaluated using Cell Counting Kit-8 (CCK-8) and 5-ethynyl2 -deoxyuridine (EdU) incorporation assays. For CCK-8 assays, 2000 indicated cells re-suspended in $100 \mu \mathrm{l}$ media were seeded into 96-well plate. After culture for 4 days, $10 \mu \mathrm{l} \mathrm{CCK-8} \mathrm{reagents} \mathrm{(Dojindo,} \mathrm{Kumamoto,}$ Japan) was added to each well. After culture for another $2 \mathrm{~h}$, the absorbance values at $450 \mathrm{~nm}$ were detected by a microplate reader (BioTek, Winooski, VT, USA) to indicate the number of viable cells. For EdU incorporation assays, indicated cells were treated with $50 \mu \mathrm{M}$ EdU (RiboBio, Guangzhou, China) for $2 \mathrm{~h}$, followed by being fixed in $4 \%$ paraformaldehyde for $30 \mathrm{~min}$ and permeabilized in $0.5 \%$ Triton X-100 for $10 \mathrm{~min}$. Then, the cells were stained using Apollo dye solution (RiboBio) and the cell nucleuses were stained using DAPI. The number of EdU-positive cells was detected by a fluorescence microscope (Carl Zeiss, Oberkochen, Germany). Cellular growth was indicated by the ratio of EdU-positive cells to all cells. Cell apoptosis was evaluated by the Caspase-3 Activity Assay Kit (Cell Signaling Technology, Danvers, MA, USA) following the manufacturer' protocol. Transwell migration and invasion assays were carried out to evaluate cell migration and invasion as we previously described [50].

\section{Mice xenograft models}

The use of mice was reviewed and approved by the Animal Ethics Committee of Affiliated Hospital of Youjiang Medical University for Nationalities. Five-week-old male BALB/C athymic nude mice were obtained from Shanghai SLAC Laboratory Animal Co. and fed in Specific Pathogen Free conditions. Luciferase-labeled SNU-398 cells with PAARH overexpression or silencing were subcutaneously injected into nude mice. When the subcutaneous xenografts grew to about $1 \mathrm{~cm}$ in diameter, they were removed and cut into small pieces, which were then transplanted into the liver of nude mice. At the 14th day after transplantation, the tumors were detected by bioluminescence imaging using IVIS ${ }^{@}$ Lumina II system (Caliper Life Sciences, Hopkinton, MA, USA). No statistical method was used to determine sample size. The experiments were not randomized. The investigators performed the bioluminescence imaging were blinded to mouse allocation.

\section{Immunohistochemistry (IHC) and terminal deoxynucleotidyl} transferase (TdT)-mediated dUTP nick end labeling (TUNEL)

The liver orthotopic xenografts were used to perform IHC staining as previously described with primary antibodies against Ki67 (\#9027, 1:400, Cell Signaling Technology) or CD31 (ab182981, 1:1000, Abcam, Cambridge, MA, USA) [49]. The liver orthotopic xenografts were also used to undertake TUNEL assays with the TUNEL Cell Apoptosis Detection Kit (Beyotime, Shanghai, China). Human HCC tissues were used to carry out IHC staining with primary antibodies against CD31 (ab9498, $1 \mu \mathrm{g} / \mathrm{ml}$, Abcam) as above described.

\section{Dual-luciferase reporter assays}

miR-6760-5p, miR-6512-3p, miR-1298-5p, miR-6720-5p, miR-4516, or miR6782-5p mimics were co-transfected with pmirGLO, pmirGLO-PAARH, or pmirGLO-HOTTIP into SNU-398 cells. Forty-eight hours after transfection, the Firefly luciferase and Renilla luciferase activities were measured using the Dual-Luciferase Reporter Assay System (Promega) following the provided protocol. pmirGLO or pmirGLO-HOTTIP was transfected into SNU-398 cells with PAARH overexpression or silencing. The luciferase activities were measured as above described $48 \mathrm{~h}$ after transfection. pGL3Basic or pGL3-VEGF promoter was co-transfected with pRL-TK (Promega) into SNU-398 cells with PAARH overexpression or silencing. The luciferase activities were measured as above described $48 \mathrm{~h}$ after transfection. pRLTK, which encodes Renilla luciferase, was used as endogenous control.

\section{RNA immunoprecipitation (RIP)}

For MS2 based RIP assays, pcDNA3.1-MS2-12X or pcDNA3.1-PAARH-MS2$12 \times$ was co-transfected with pMS2-GFP (Addgene) into SNU-398 cells. Forty-eight hours after transfection, the cells were used to undertake RIP assays with a GFP antibody (11814460001, $5 \mu \mathrm{g}$ per reaction, Roche) and the Magna RIP RNA-Binding Protein Immunoprecipitation Kit (Millipore) following the manufacturer's protocol. To detect the RNAs binding to HIF$1 \mathrm{a}$, SNU-398 cells were cultured in hypoxia $\left(1 \% \mathrm{O}_{2}\right)$ for $24 \mathrm{~h}$. Then, the cells were used to undertake RIP assays with a HIF-1a antibody (NB100-105, $5 \mu \mathrm{g}$ per reaction, Novus, Littleton, CO, USA) and the Magna RIP RNABinding Protein Immunoprecipitation Kit. The enriched RNAs were measured using qRT-PCR as above described.

\section{RNA pull-down}

PAARH was in vitro transcribed from pSPT19-PAARH using the MEGAscript ${ }^{\circ}$ Kit (Thermo Fisher) with T7 RNA polymerase. PAARH was labeled using the Pierce ${ }^{\text {TM }}$ RNA $3^{\prime}$ End Desthiobiotinylation Kit (Thermo Scientific). SNU-398 cells were cultured in hypoxia $\left(1 \% \mathrm{O}_{2}\right)$ for $24 \mathrm{~h}$. Then, the cells were used to undertake RNA pull-down assays with the Pierce ${ }^{m}$ Magnetic RNA-Protein Pull-Down Kit (Thermo Scientific). The RNA present in the pull-down material was measured using qRT-PCR as above described, and the protein present in the pull-down material was measured by western blot.

\section{Western blot}

Total protein present in the pull-down material was separated by sodium dodecyl sulfate-polyacrylamide gel electrophoresis, followed by being transferred onto polyvinylidene fluoride membrane. After being blocked in $5 \%$ skimmed milk, the membranes were incubated with primary antibodies against HIF-1a (NB100-105, 1:500, Novus) or GAPDH (ab8245, 1:5000, Abcam). The second antibody used was IRDye 680RD Goat antiMouse IgG (Li-Cor, Lincoln, NE, USA). Lastly, the membranes were scanned on an Odyssey infrared scanner (Li-Cor).

\section{In vitro tube formation}

Indicated HCC cells were cultured in normoxia or hypoxia $\left(1 \% \mathrm{O}_{2}\right)$ for $24 \mathrm{~h}$. Then, the supernatant was collected to be used as conditioned medium (CM) for HUVEC. Each well of prechilled 24-well plates was coated with $100 \mu$ l Matrigel (BD Biosciences, Franklin Lakes, NJ, USA) and incubated at $37^{\circ} \mathrm{C}$ for $1 \mathrm{~h}$. In total, $2 \times 10^{4}$ HUVEC re-suspended in above-described CM were seeded into the solidified gel. After culture for $16 \mathrm{~h}$, the endothelial tubes were counted under photomicroscope.

\section{Enzyme linked immunosorbent assay (ELISA)}

VEGF concentration in cell culture supernatant was measured by ELISA using the Human VEGF Quantikine ELISA Kit (R\&D Systems, Minneapolis, MN, USA) following the manufacturer's protocol. The absorbance values were measured by a microplate reader (BioTek). Serial dilution of human recombinant VEGF was performed in each assay to draw a standard curve.

\section{Chromatin immunoprecipitation (ChIP)}

Indicated HCC cells were cultured in normoxia or hypoxia $\left(1 \% \mathrm{O}_{2}\right)$ for $24 \mathrm{~h}$. Then, the cells were used to carry out ChIP assays with the EZ-Magna ChIP A/G (17-10086, Millipore) and a HIF-1a antibody (NB100-105, $5 \mu \mathrm{g}$ per reaction, Novus) following the provided protocol. The enriched DNA was measured using qRT-PCR with the primers $5^{\prime}$-CTGGCGGGTAGGTTTGAAT C-3' (sense) and $5^{\prime}$-AGAACGGGAAGCTGTGTGG-3' (anti-sense) to detect VEGF promoter.

\section{Statistical analysis}

GraphPad Prism 6.0 Software was employed to perform all statistical analyses. Wilcoxon matched-pairs signed rank test, log-rank test, Student's 
$t$ test, one-way ANOVA followed by Dunnett's multiple comparisons test, Mann-Whitney test, Kruskal-Wallis test followed by Dunn's multiple comparisons test, and Pearson chi-square test were undertaken as shown in the figure and table legends. $p<0.05$ was considered statistically significant.

\section{RESULTS}

PAARH was highly expressed and correlated with advanced stage, vascular invasion, and poor survival in HCC

RNA-seq data of TCGA LIHC revealed that PAARH (CMB9-22P13.1) was highly expressed in HCC tissues compared with normal liver tissues (Fig. 1a), analyzed by GEPIA (http://gepia.cancer-pku.cn/ index.html) [44]. RNA-seq data of TCGA LIHC also revealed that high expression of PAARH was associated with worse overall survival and disease-free survival (Fig. 1b, c). Furthermore, we randomly collected 72 pairs of HCC tissues and matched adjacent noncancerous liver tissues. qRT-PCR results confirmed the increased expression of PAARH in HCC tissues (Fig. 1d). Analyses of the correlations between PAARH expression and clinicopathological characteristics in these $72 \mathrm{HCC}$ cases revealed that high PAARH expression was correlated with advanced Barcelona Clinic Liver Cancer (BCLC) stage, poor differentiation, and microvascular invasion (Supplementary Table 1). Kaplan-Meier survival analyses in these $72 \mathrm{HCC}$ cases also revealed that high expression of PAARH was associated with worse overall survival and disease-free survival (Fig. 1e, f). Three in silico tools, CPAT (http://lilab. research.bcm.edu/), CPC 2.0 (http://cpc2.gao-lab.org/), and TestCode (https://www.bioinformatics.org/sms/testcode.html) all indicated PAARH as a non-coding RNA (Supplementary Fig. 1a-c).
Biochemical fractionation of SNU-398 cells followed by qRT-PCR revealed that PAARH was distributed in both cytoplasm and nucleus (Supplementary Fig. 1d). These data demonstrated that PAARH was a highly expressed IncRNA in HCC. High expression of PAARH was correlated with advanced stage, poor differentiation, microvascular invasion, poor overall survival, and poor diseasefree survival.

PAARH facilitated HCC cellular growth, migration, and invasion, and inhibited HCC cellular apoptosis

Given the clinical relevance of PAARH in HCC, we further investigated the potential biological roles of PAARH in HCC. SNU-398 and SK-HEP-1 cells with PAARH stable overexpression were constructed via transfection of PAARH overexpression plasmid (Fig. 2a). CCK-8 and EdU incorporation assays presented that ectopic expression of PAARH facilitated HCC cellular growth (Fig. 2b, c). Caspase-3 activity assays presented that ectopic expression of PAARH inhibited HCC cellular apoptosis (Fig. 2d). Transwell migration and invasion experiments presented that ectopic expression of PAARH facilitated HCC cellular migration and invasion (Fig. 2e, f). These data demonstrated that ectopic expression of PAARH facilitated HCC cellular growth, migration, and invasion, and inhibited HCC cellular apoptosis.

PAARH silencing restricted HCC cellular growth, migration, and invasion, and promoted HCC cellular apoptosis

To completely explore the potential biological roles of PAARH in HCC, SNU-398 and Huh7 cells with PAARH stable silencing were constructed via infection of two independent PAARH specific a
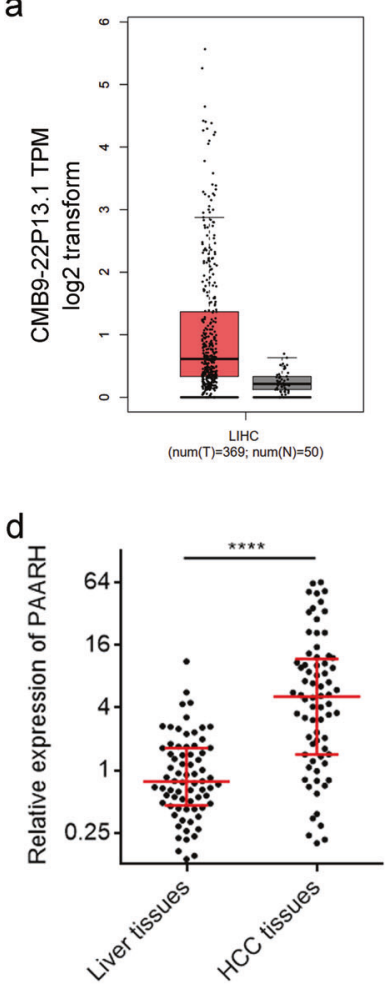

b

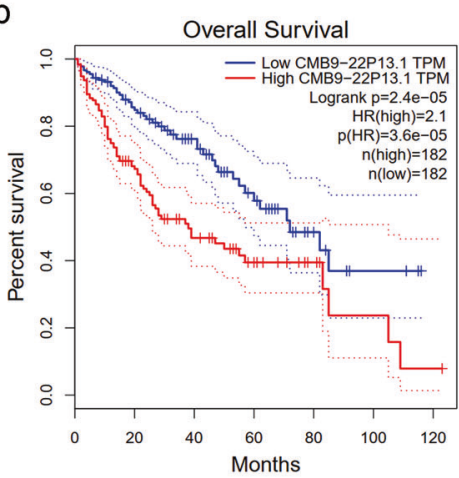

e

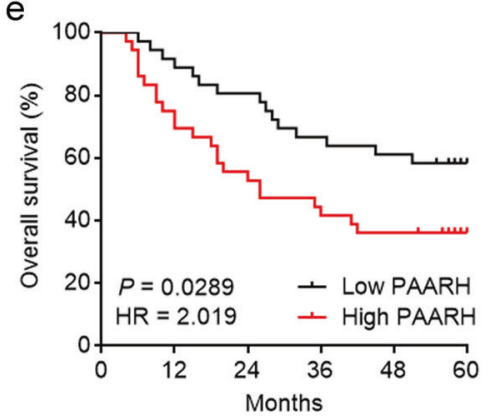

C

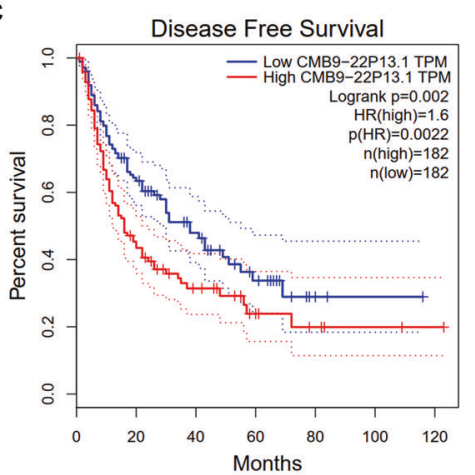

f

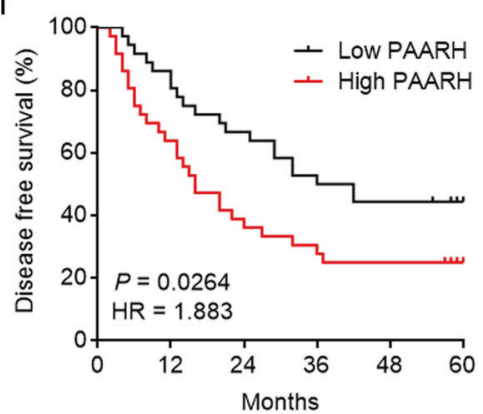

Fig. 1 PAARH was highly expressed and correlated with poor survival in HCC. a PAARH (CMB9-22P13.1) expression in 369 HCC tissues and 50 liver tissues according to TCGA LIHC dataset, analyzed by GEPIA. $\mathbf{b}$ The correlation between PAARH (CMB9-22P13.1) expression and overall survival according to TCGA LIHC dataset, analyzed by GEPIA. c The correlation between PAARH (CMB9-22P13.1) expression and disease-free survival according to TCGA LIHC dataset, analyzed by GEPIA. d PAARH expression in 72 pairs of HCC tissues and adjacent noncancerous liver tissues was measured by qRT-PCR. ${ }^{* * *} p<0.0001$ by Wilcoxon matched-pairs signed rank test. e Kaplan-Meier survival analysis of the correlation between PAARH expression and overall survival in our HCC cohort. $n=72, p=0.0289$, HR $=2.019$ by log-rank test. f Kaplan-Meier survival analysis of the correlation between PAARH expression and disease-free survival in our HCC cohort. $n=72, p=0.0264, \mathrm{HR}=1.883$ by log-rank test. 

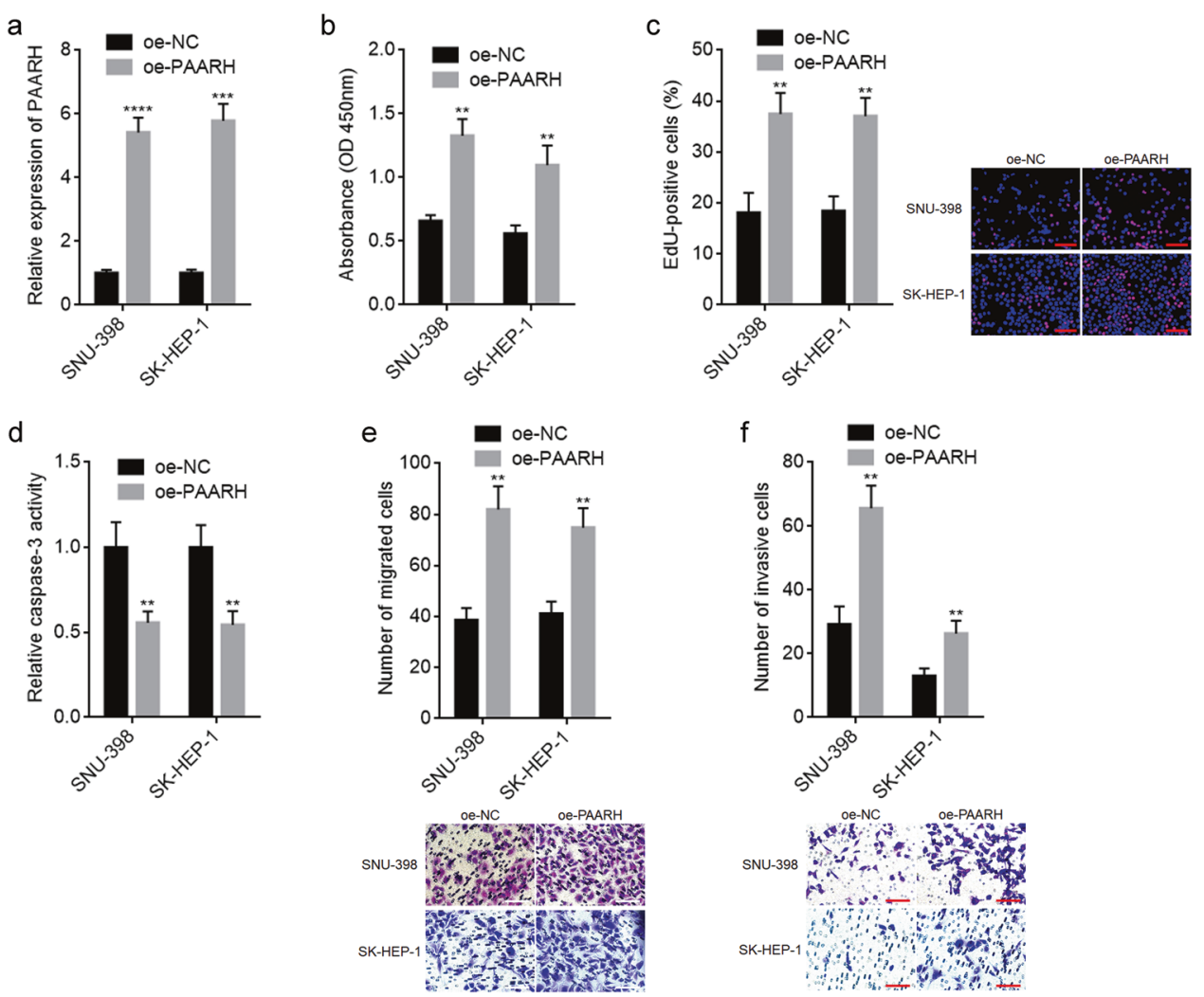

Fig. 2 Ectopic expression of PAARH enhanced HCC cellular malignant phenotype in vitro. a PAARH expression in SNU-398 and SK-HEP-1 cells with PAARH stable overexpression was measured by qRT-PCR. $\mathbf{b}$ Cell growth of SNU-398 and SK-HEP-1 cells with PAARH overexpression was detected using CCK-8 assays. c Cell growth of SNU-398 and SK-HEP-1 cells with PAARH overexpression was detected using EdU incorporation assays. Scale bars, $100 \mu \mathrm{m}$. d Cell apoptosis of SNU-398 and SK-HEP-1 cells with PAARH overexpression was detected using caspase-3 activity assays. e Cell migration of SNU-398 and SK-HEP-1 cells with PAARH overexpression was detected using transwell migration assays. Scale bars, $100 \mu \mathrm{m}$. f Cell invasion of SNU-398 and SK-HEP-1 cells with PAARH overexpression was detected using transwell invasion assays. Scale bars, $100 \mu \mathrm{m}$. Results are shown as mean \pm SD based on three independent experiments. ${ }^{* *} p<0.01,{ }^{* * *} p<0.001,{ }^{* * * *} p<0.0001$ by Student's $t$ test.

shRNA lentiviruses (Fig. 3a). CCK-8 and EdU incorporation assays presented that PAARH silencing restricted HCC cellular growth (Fig. 3b, c). Caspase-3 activity assays presented that PAARH silencing promoted HCC cellular apoptosis (Fig. 3d). Transwell migration and invasion experiments presented that PAARH silencing restricted HCC cellular migration and invasion (Fig. 3e, f). These data demonstrated that PAARH silencing restricted HCC cellular growth, migration, and invasion, and promoted HCC cellular apoptosis.

\section{PAARH facilitated HCC tumor progression in vivo}

To further explore the potential roles of PAARH in mouse xenograft models, small pieces of subcutaneous tumors formed by luciferase-labeled SNU-398 cells with PAARH stable overexpression or silencing were orthotopically transplanted into the livers of nude mice. Bioluminescence imaging showed that the xenografts derived from PAARH overexpressed SNU-398 cells were significantly larger than those derived from control SNU-398 cells (Fig. 4a). The xenografts derived from PAARH silenced SNU-398 cells were remarkably smaller than those derived from control SNU-398 cells (Fig. 4b). Proliferation marker Ki67 IHC staining of orthotropic xenografts revealed that the xenografts derived from PAARH overexpressed SNU-398 cells had more Ki67 positive cells than those derived from control SNU-398 cells (Fig. 4c). Conversely, the xenografts derived from PAARH silenced SNU398 cells had less Ki67 positive cells than those derived from control SNU-398 cells (Fig. 4d). Apoptosis marker TUNEL staining of orthotropic xenografts revealed that the xenografts derived from PAARH overexpressed SNU-398 cells had less apoptotic cells than those derived from control SNU-398 cells (Fig. 4e). Conversely, the xenografts derived from PAARH silenced SNU398 cells had more apoptotic cells than those derived from control SNU-398 cells (Fig. 4f). In addition, CD31 IHC staining orthotropic xenografts revealed that the xenografts derived from PAARH overexpressed SNU-398 cells had increased microvessel density (MVD) compared with those derived from control SNU-398 cells (Fig. 4g). Conversely, the xenografts derived from PAARH silenced SNU-398 cells had decreased MVD compared with those derived from control SNU-398 cells (Fig. 4h). These data demonstrated that PAARH also exerted oncogenic roles in vivo. PAARH not only facilitated HCC cellular malignant phenotype, but also promoted angiogenesis.

\section{PAARH upregulated HOTTIP via sponging miRNAs}

To explore the mechanisms underlying the oncogenic roles of PAARH in HCC, we searched the genes whose expressions are correlated with PAARH in HCC tissues based on TCGA LIHC dataset analyzed by GEPIA. HOTTIP ranked the second with Pearson correlation coefficient of 0.56 (Fig. 5a). The positive correlation between HOTTIP and PAARH expression was further verified in our HCC cohort (Fig. 5b). HOTTIP is a well-known oncogenic IncRNA in various malignancies, including HCC [51-53]. HOTTIP expression in PAARH overexpressed and silenced HCC cells was measured by qRT-PCR. As shown in Fig. 5c, d, HOTTIP was significantly increased in SNU-398 and SK-HEP-1 cells after PAARH overexpression, and significantly reduced in SNU-398 and Huh7 cells after PAARH silencing. Due to PAARH was distributed in both cytoplasm and nucleus. Many cytoplasmic IncRNAs were revealed to function as 

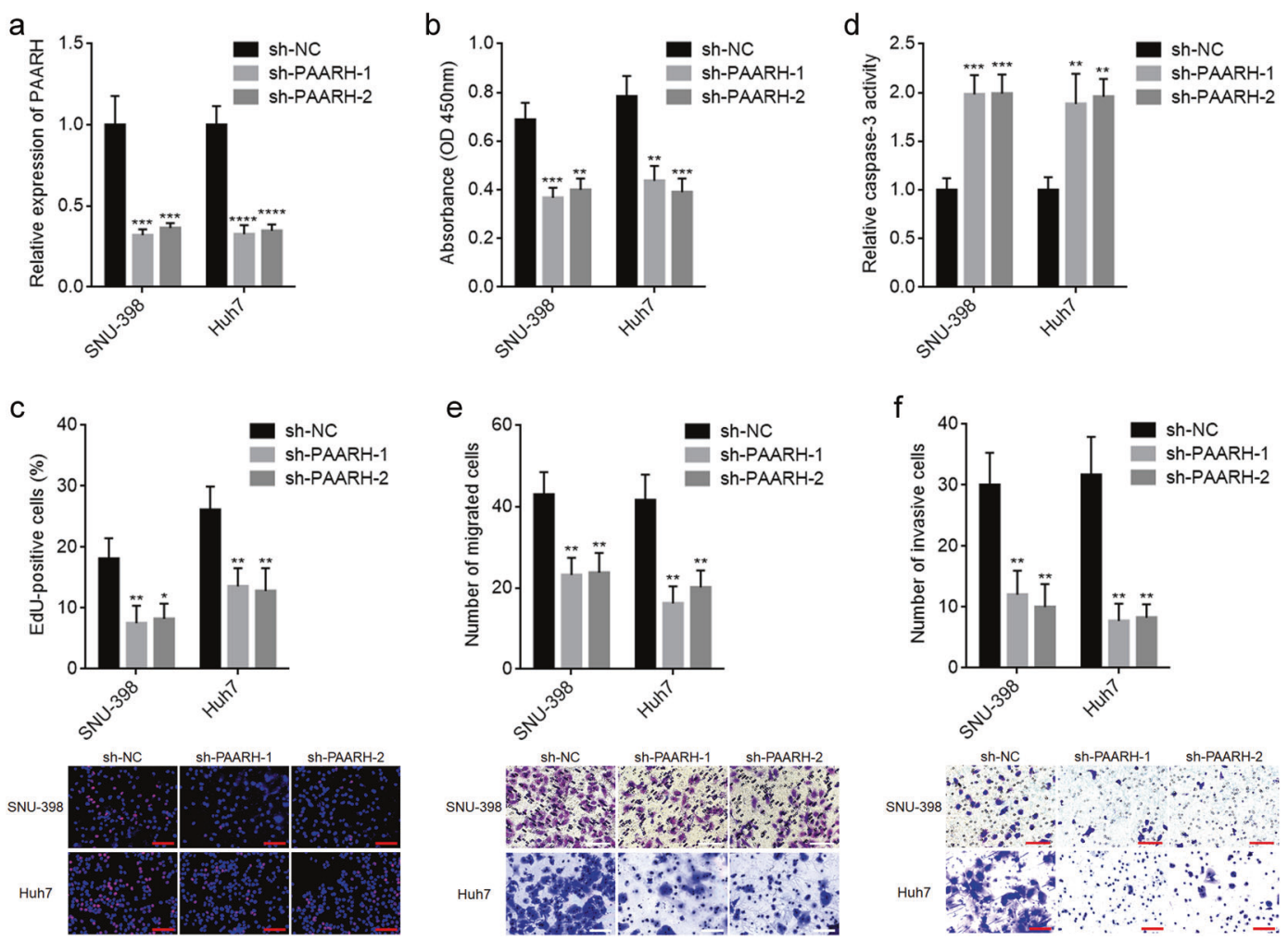

Fig. 3 PAARH silencing reduced HCC cellular malignant phenotype in vitro. a PAARH expression in SNU-398 and Huh7 cells with PAARH stable silencing was measured by qRT-PCR. b Cell growth of SNU-398 and Huh7 cells with PAARH silencing was detected using CCK-8 assays. $\mathbf{c}$ Cell growth of SNU-398 and Huh7 cells with PAARH silencing was detected using EdU incorporation assays. Scale bars, $100 \mu$ m. d Cell apoptosis of SNU-398 and Huh7 cells with PAARH silencing was detected using caspase-3 activity assays. e Cell migration of SNU-398 and Huh7 cells with PAARH silencing was detected using transwell migration assays. Scale bars, $100 \mu$ m. $\mathbf{f}$ Cell invasion of SNU-398 and Huh7 cells with PAARH silencing was detected using transwell invasion assays. Scale bars, $100 \mu \mathrm{m}$. Results are shown as mean \pm SD based on three independent experiments. ${ }^{*} p<0.05,{ }^{* *} p<0.01,{ }^{* * *} p<0.001,{ }^{* * *} p<0.0001$ by one-way ANOVA followed by Dunnett's multiple comparisons test.

competing endogenous RNA (ceRNA) via sponging common miRNAs and relieving the repressive roles of miRNAs on their targets [33, 38]. To explore whether PAARH increased HOTTIP expression through the ceRNA mechanism, the miRNAs binding to PAARH and HOTTIP were predicted by LncBase Predicted v.2 (http://carolina.imis.athena-innovation.gr/diana_tools/web/index. php? $r=$ Incbasev2\%2Findex-predicted). Intriguingly, miR-6760-5p, miR-6512-3p, miR-1298-5p, miR-6720-5p, miR-4516, and miR-6782$5 p$ were predicted to bind both PAARH and HOTTIP (Supplementary Table 2). Dual-luciferase reporter assays presented that miR6760-5p, miR-6512-3p, miR-1298-5p, miR-6720-5p, miR-4516, and miR-6782-5p targeted both PAARH and HOTTIP (Fig. 5e). To explore whether PAARH binds these miRNAs, MS2 based RIP assays were performed as previously described [33]. The results revealed that miR-6760-5p, miR-6512-3p, miR-1298-5p, miR-6720$5 p$, miR-4516, and miR-6782-5p were specially enriched in MS2PAARH group (Fig. 5f). miR-155-5p was used as NC. To further confirm the binding of these miRNAs to PAARH, RNA pull-down experiments using RNA end-labeled with desthiobiotin were undertaken. As shown in Fig. 5g, miR-6760-5p, miR-6512-3p, miR-1298-5p, miR-6720-5p, miR-4516, and miR-6782-5p, but not miR-155-5p, were specifically enriched by PAARH, but not by negative RNA control [poly $(A)_{25}$ RNA]. These results suggested that PAARH bound miR-6760-5p, miR-6512-3p, miR-1298-5p, miR-6720$5 p, m i R-4516$, and miR-6782-5p. Dual-luciferase reporter assays presented that conversely with these miRNAs, ectopic expression of PAARH increased the luciferase activities of the constructed reporter containing HOTTIP (Fig. 5h). PAARH silencing reduced the luciferase activities of the constructed reporter containing HOTTIP
(Fig. 5i). To further investigate whether PAARH regulates HOTTIP in a miRNA-dependent manner, DICER was knocked known to repress miRNAs biogenesis. At the condition of DICER depletion, the upregulation of HOTTIP by PAARH was largely abolished (Fig. 5j, compared with Fig. 5c). Similarly, at the condition of DICER depletion, the downregulation of HOTTIP by PAARH silencing was also largely abolished (Fig. 5k, compared with Fig. 5d). Collectively, these data demonstrated that PAARH upregulated HOTTIP via sponging miR-6760-5p, miR-6512-3p, miR-1298-5p, miR-6720-5p, miR-4516, and miR-6782-5p.

\section{Depletion of HOTTIP largely reversed the roles of PAARH in modulating HCC cellular growth, apoptosis, migration, and invasion}

Given that HOTTIP was identified as a downstream target of PAARH, we further investigated whether HOTTIP is a critical mediator of the oncogenic roles of PAARH in HCC. HOTTIP was stably depleted in PAARH overexpressed SNU-398 cells via infection of HOTTIP specific shRNA lentiviruses (Fig. 6a). CCK-8 and EdU incorporation assays presented that depletion of HOTTIP largely abolished PAARH-induced promotion of cellular growth (Fig. 6b, c). Caspase-3 activity assays presented that depletion of HOTTIP largely abolished PAARH-induced repression of apoptosis (Fig. 6d). Transwell migration and invasion experiments presented that depletion of HOTTIP largely abolished PAARH-induced promotion of migration and invasion (Fig. 6e, f). These data demonstrated that PAARH exerted oncogenic roles in HCC cellular growth, apoptosis, migration, and invasion largely through upregulating HOTTIP. 

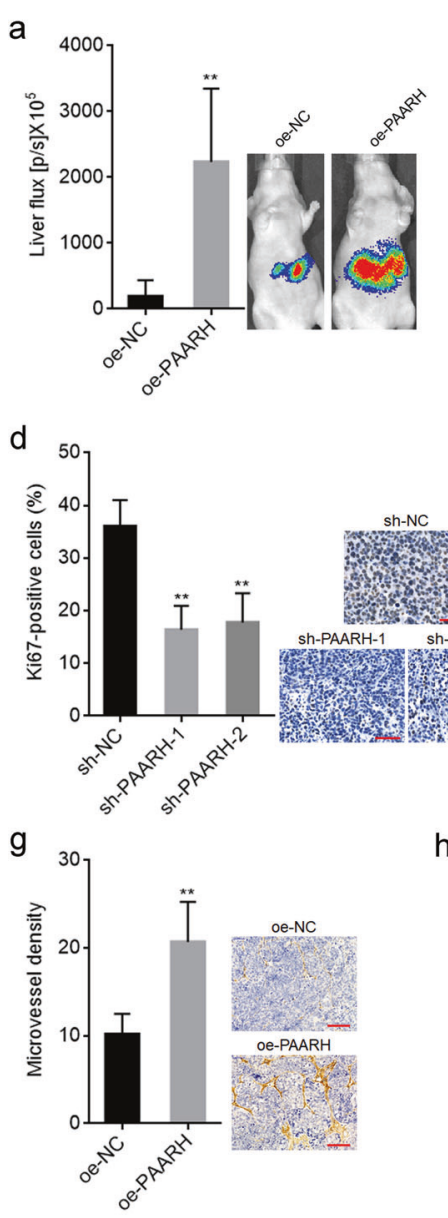
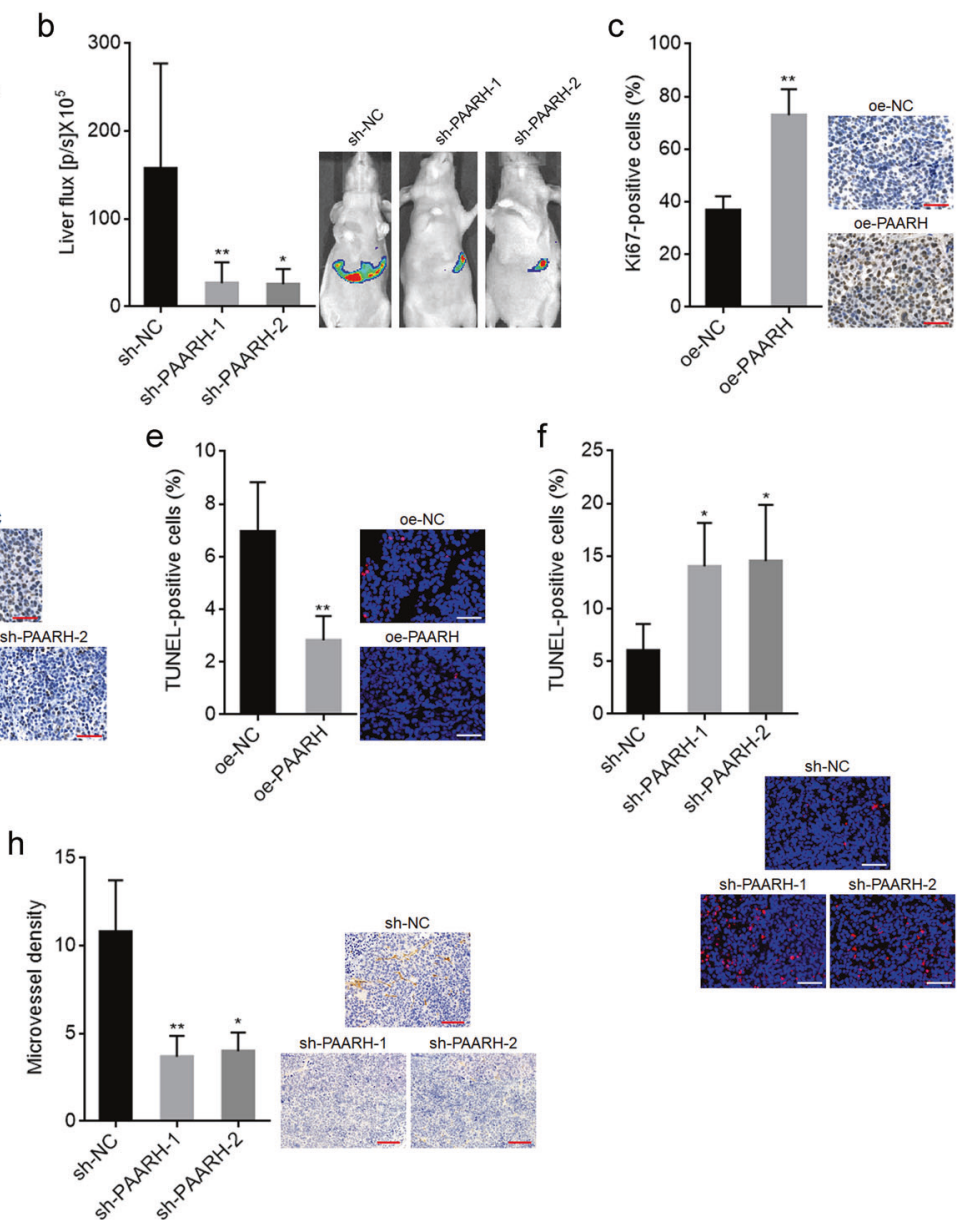

f

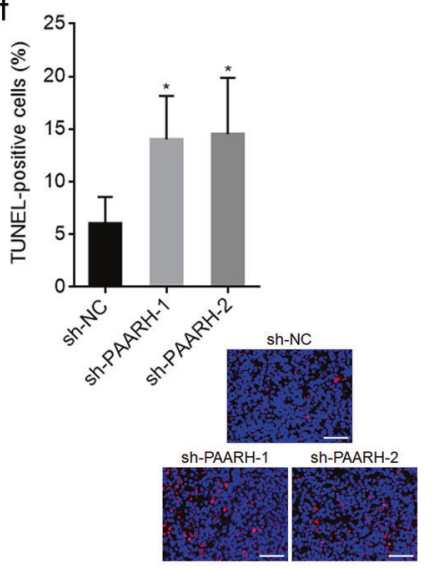

Fig. 4 PAARH facilitated HCC progression in vivo. a Bioluminescence imaging of liver tumors in mice at day 14 after inoculation with small pieces of subcutaneous tumors formed by luciferase-labeled SNU-398 cells with PAARH stable overexpression or control. b Bioluminescence imaging of liver tumors in mice at day 14 after inoculation with small pieces of subcutaneous tumors formed by luciferase-labeled SNU-398 cells with PAARH stable silencing or control. c Ki67 IHC staining of liver tumors formed by SNU-398 cells with PAARH stable overexpression or control. Scale bars, $50 \mu \mathrm{m}$. d Ki67 IHC staining of liver tumors formed by SNU-398 cells with PAARH stable silencing or control. Scale bars, $50 \mu \mathrm{m}$. e TUNEL staining of liver tumors formed by SNU-398 cells with PAARH stable overexpression or control. Scale bars, $50 \mu \mathrm{m}$. $\mathbf{f}$ TUNEL staining of liver tumors formed by SNU-398 cells with PAARH stable silencing or control. Scale bars, $50 \mu \mathrm{m}$. g Microvessel density of liver tumors formed by SNU-398 cells with PAARH stable overexpression or control was detected using CD31 IHC staining. Scale bars, $100 \mu \mathrm{m}$. $\mathbf{h}$ Microvessel density of liver tumors formed by SNU-398 cells with PAARH stable silencing or control was detected using CD31 IHC staining. Scale bars, $100 \mu \mathrm{m}$. Results are shown as mean \pm SD based on $n=6$ mice in each group. ${ }^{*} p<0.05,{ }^{* *} p<0.01$ by Mann-Whitney test (a, c, e, $\left.\mathbf{g}\right)$ or Kruskal-Wallis test followed by Dunn's multiple comparisons test $(\mathbf{b}, \mathbf{d}, \mathbf{f}, \mathbf{h})$.

\section{PAARH facilitated angiogenesis via upregulating VEGF}

Given that PAARH expression was positively correlated with vascular invasion in HCC tissues and PAARH promoted angiogenesis in xenografts (Fig. 4g, h), we further explored the influences of PAARH on angiogenesis and the underlying mechanism. CM from PAARH overexpressed and silenced HCC cells were collected to stimulate HUVEC cells and the tubeforming abilities of HUVEC cells were detected. Under normoxia, CM from SNU-398 and SK-HEP-1 cells with PAARH overexpression did not have significant effects on tube-forming abilities of HUVEC cells (data not shown). Given that hypoxia is well-known to be involved in various malignancies, including HCC [54]. Thus, we further investigated the potential effects of PAARH on angiogenesis under hypoxia. CM from SNU-398 and SK-HEP-1 cells with PAARH overexpression significantly increased tubeforming abilities of HUVEC cells compared with CM from control SNU-398 and SK-HEP-1 cells under hypoxia (Fig. 7a). Conversely, CM from SNU-398 and Huh7 cells with PAARH silencing significantly repressed tube-forming abilities of HUVEC cells under hypoxia (Fig. 7b). VEGF is a well-known critical angiogenetic factor $[55,56]$. Thus, we further investigated whether VEGF is a downstream target of PAARH in HCC. VEGF expression was significantly increased in SNU-398 and SK-HEP-1 cells after PAARH overexpression under hypoxia (Fig. 7c). Under normoxia, PAARH overexpression did not regulate VEGF (Supplementary Fig. 2a). Conversely, VEGF expression was significantly reduced in SNU-398 and Huh7 cells after PAARH silencing under hypoxia (Fig. 7d). PAARH silencing did not regulate VEGF under normoxia (Supplementary Fig. 2b). ELISA revealed that SNU-398 and SKHEP-1 cells with PAARH overexpression had increased VEGF secretion compared with control cells under hypoxia (Fig. 7e). SNU-398 and Huh7 cells with PAARH silencing had reduced VEGF secretion compared with control cells under hypoxia (Fig. 7f). The expression of VEGF was significantly positively correlated with PAARH in HCC tissues based on TCGA LIHC dataset analyzed by GEPIA (Fig. 7g). Furthermore, the positive correlation between VEGF and PAARH expression in HCC tissues was further verified in our HCC cohort (Fig. 7h). CD31 IHC staining was performed in HCC tissues. The results revealed that PAARH was significantly upregulated in HCC tissues with high MVD compared with those with low MVD (Fig. 7i), supporting the roles of PAARH in promoting angiogenesis in vivo. 


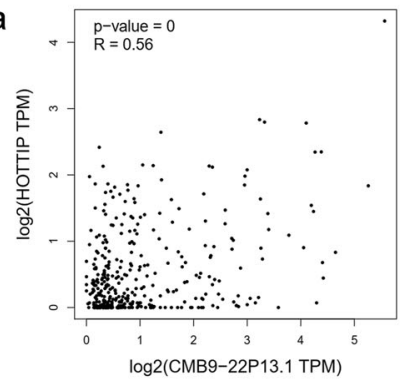

d

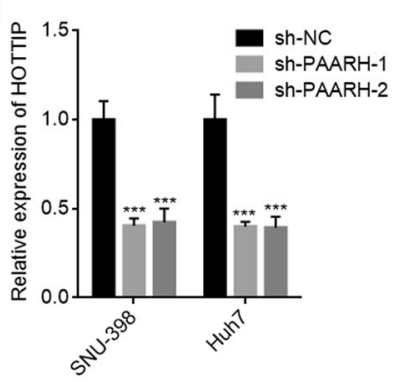

f
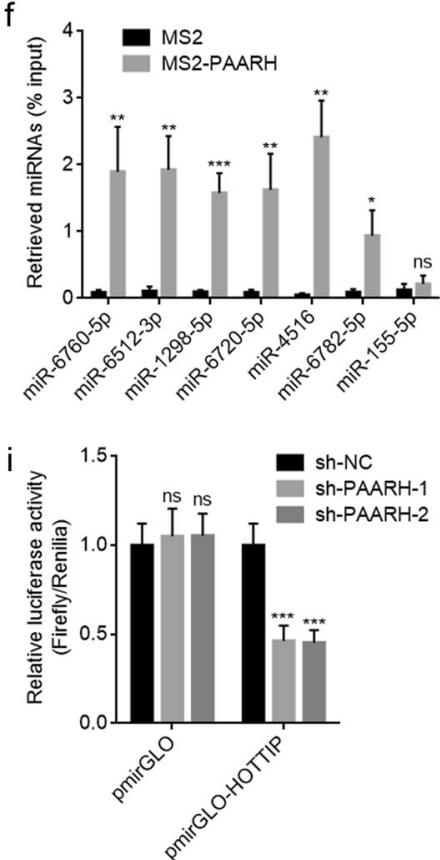

b

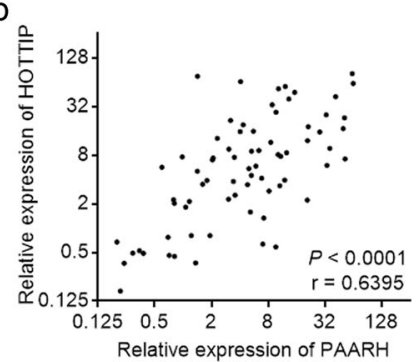

e

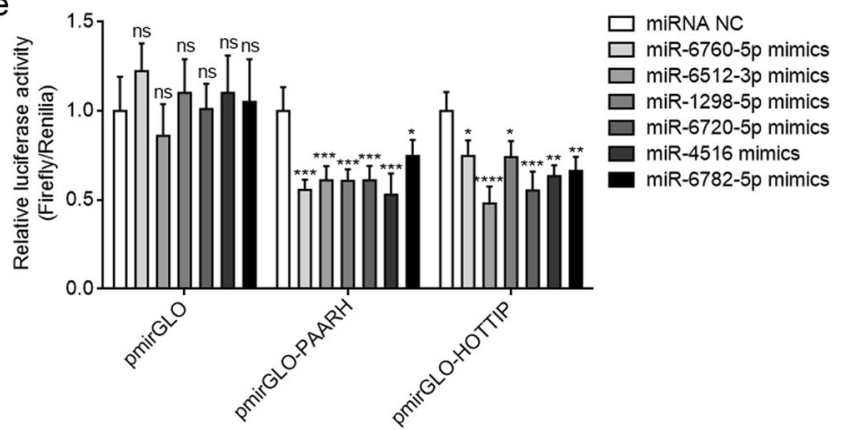

g

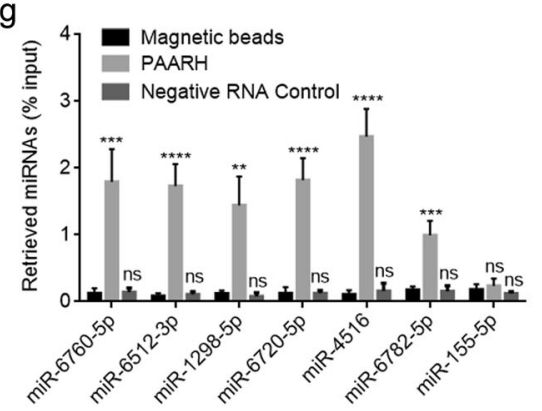

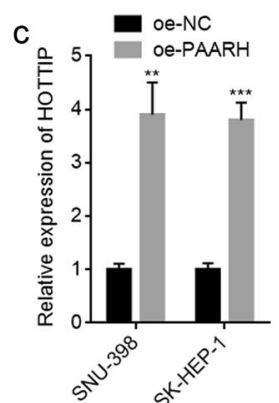

h

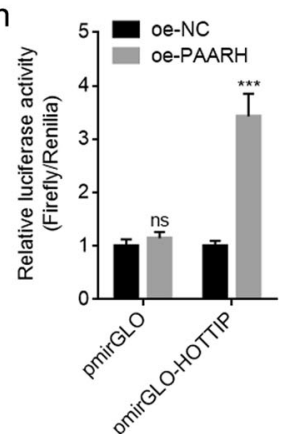

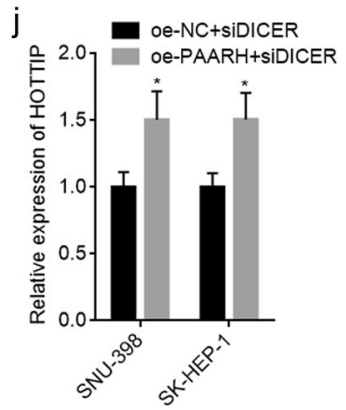

k

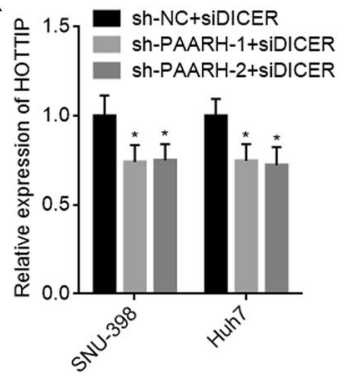

Fig. 5 PAARH increased HOTTIP expression via functioning as a ceRNA. a The correlation between HOTTIP and PAARH (CMB9-22P13.1) expression in HCC tissues according to TCGA LIHC dataset, analyzed by GEPIA. b The correlation between HOTTIP and PAARH expression in our HCC cohort. $n=72, p<0.0001, r=0.6395$ by Spearman correlation analysis. c HOTTIP expression levels in SNU-398 and SK-HEP-1 cells with PAARH overexpression or control were measured by qRT-PCR. d HOTTIP expression levels in SNU-398 and Huh7 cells with PAARH stable silencing or control was measured by qRT-PCR. e Luciferase activity in SNU-398 cells co-transfected with miR-6760-5p, miR-6512-3p, miR-1298$5 p$, miR-6720-5p, miR-4516, or miR-6782-5p and luciferase reporters containing nothing, PAARH or HOTTIP. Results are shown as the relative ratio of firefly luciferase activity to renilla luciferase activity. $\mathbf{f}$ MS2 based RIP assays followed by qRT-PCR to detect miRNAs bound to PAARH. g RNA pull-down experiments using RNA end-labeled with desthiobiotin, followed by qRT-PCR to detect miRNAs bound to PAARH. h Luciferase activity in SNU-398 cells with PAARH overexpression or control after transfection of luciferase reporters containing nothing or HOTTIP. Results are shown as the relative ratio of firefly luciferase activity to renilla luciferase activity. i Luciferase activity in SNU-398 cells with PAARH silencing or control after transfection of luciferase reporters containing nothing or HOTTIP. Results are shown as the relative ratio of firefly luciferase activity to renilla luciferase activity. j HOTTIP expression levels in SNU-398 and SK-HEP-1 cells with PAARH overexpression or control after transfection of DICER siRNA pool were measured by qRT-PCR. $\mathbf{k}$ HOTTIP expression levels in SNU-398 and Huh7 cells with PAARH stable silencing or control after transfection of DICER siRNA pool was measured by qRT-PCR. For c-k, results are shown as mean \pm SD based on three independent experiments. ${ }^{*} p<0.05,{ }^{* *} p<0.01,{ }^{* * *} p<0.001,{ }^{* * * *} p<0.0001$, ns not significant by Student's $t$ test (c, f, $\left.\mathbf{h}, \mathbf{j}\right)$ or one-way ANOVA followed by Dunnett's multiple comparisons test $(\mathbf{d}, \mathbf{e}, \mathbf{g}, \mathbf{i}, \mathbf{k})$. 
a

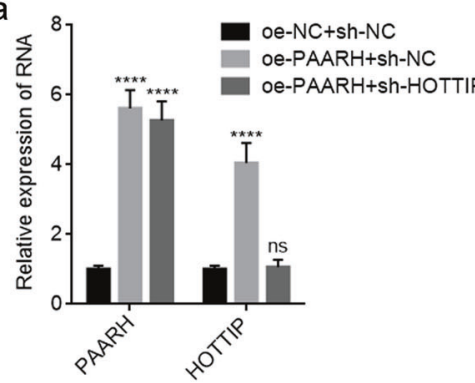

b

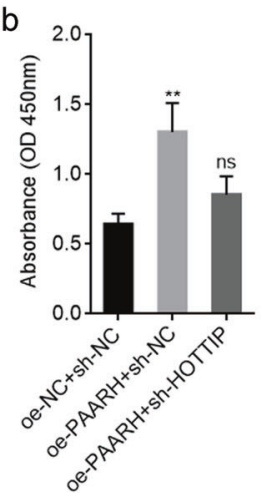

d

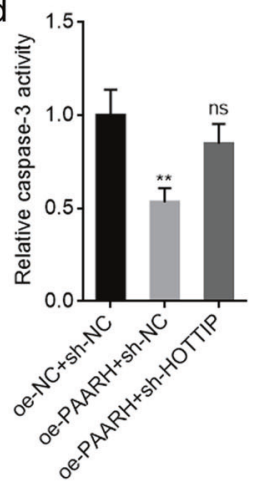

e

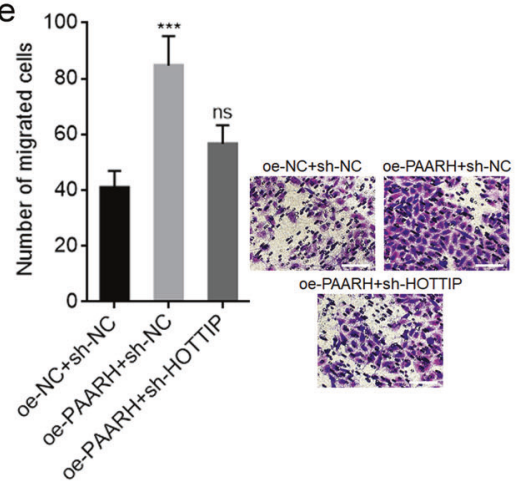

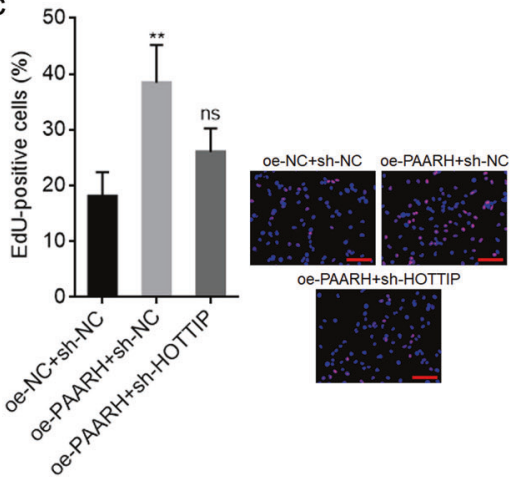

f

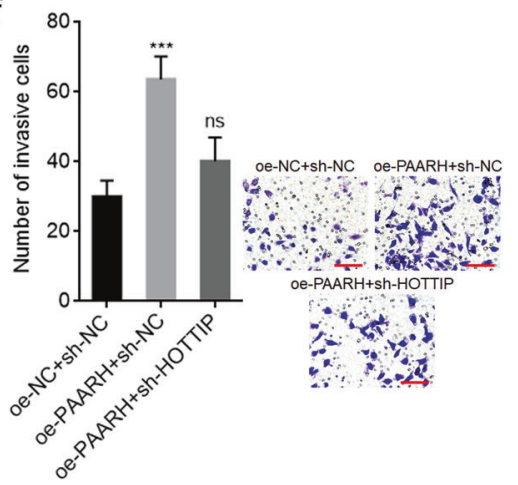

Fig. 6 Depletion of HOTTIP reversed the oncogenic roles of PAARH in HCC. a PAARH and HOTTIP expression in SNU-398 cells with PAARH overexpression and concurrent HOTTIP depletion. b Cell growth of SNU-398 cells with PAARH overexpression and HOTTIP depletion was detected using CCK-8 assays. c Cell growth of SNU-398 cells with PAARH overexpression and HOTTIP depletion was detected using EdU incorporation assays. Scale bars, $100 \mu \mathrm{m}$. d Cell apoptosis of SNU-398 cells with PAARH overexpression and HOTTIP depletion was detected using caspase-3 activity assays. e Cell migration of SNU-398 cells with PAARH overexpression and HOTTIP depletion was detected using transwell migration assays. Scale bars, $100 \mu \mathrm{m}$. f Cell invasion of SNU-398 cells with PAARH overexpression and HOTTIP depletion was detected using transwell invasion assays. Scale bars, $100 \mu \mathrm{m}$. Results are shown as mean \pm SD based on three independent experiments. ${ }^{* *} p<0.01,{ }^{* * *} p$ $<0.001,{ }^{* * * *} p<0.0001$, ns not significant, by one-way ANOVA followed by Dunnett's multiple comparisons test.

PAARH facilitated HIF-1a-induced transcription of VEGF via prompting the recruitment of HIF-1a to VEGF promoter Under hypoxia, Hypoxia Inducible Factor-1 Subunit Alpha (HIF1a) was induced and further activated VEGF expression [57]. Therefore, we investigated whether HIF-1a participates in the modulation of VEGF by PAARH. PAARH was predicted to bind HIF-1a by RNA-Protein Interaction Prediction (RPISeq) (http:// pridb.gdcb.iastate.edu/RPISeq/index.html). RIP assays presented that PAARH was specifically enriched in HIF-1a antibody group (Fig. 8a). RNA pull-down assays using PAARH end-labeled with desthiobiotin presented that HIF-1a was specifically enriched by PAARH, but not by negative RNA control [poly $(A)_{25}$ RNA] (Fig. 8b). These data demonstrated that PAARH directly bound to HIF-1a. ChIP assays revealed that ectopic expression of PAARH increased the binding of HIF-1a to VEGF promoter under hypoxia, but not under normoxia (Fig. 8c). Consistently, PAARH silencing reduced the binding of HIF-1a to VEGF promoter under hypoxia, but not under normoxia (Fig. 8d). These data demonstrated that PAARH facilitated the recruitment of HIF-1a to VEGF promoter under hypoxia. Dual-luciferase reporter assays showed that ectopic expression of PAARH increased VEGF promoter activity under hypoxia (Fig. 8e). PAARH silencing decreased VEGF promoter activity under hypoxia (Fig. 8f). Depletion of HIF-1a abolished PAARH-induced upregulation of VEGF under hypoxia (Fig. 8g). Furthermore, Depletion of VEGF also abolished PAARH-induced increasing of tube-forming abilities of HUVEC cells under hypoxia (Fig. 8h). Collectively, these data demonstrated that PAARH physically bound HIF-1a, promoted the recruitment of HIF-1a to VEGF promoter, and activated VEGF transcription.

\section{DISCUSSION}

HCC is recognized as the second most lethal cancer, only after pancreatic cancer $[2,58]$. The extremely poor prognosis of HCC indicated that further uncovering the molecular pathogenesis of HCC and developing more efficient systemic therapies are urgently needed.

Here, we identified a novel oncogenic IncRNA PAARH in HCC. $P A A R H$ is located at chromosome 11q13.1 and has three exons. Both the TCGA LIHC dataset and our own HCC cohort revealed that PAARH is highly expressed in HCC tissues compared with noncancerous liver tissues. Furthermore, both data revealed that high PAARH expression is correlated with poor overall survival and disease-free survival. Thus, PAARH is a potential prognosis biomarker for HCC, which needs further multi-central investigations. In addition, high expression of PAARH was also revealed to be positively associated with BCLC stage, poor differentiation, and microvascular invasion. These clinical relevancies implied that PAARH may exert regulatory roles in HCC.

Gain-of and loss-of function assays revealed that PAARH facilitated HCC cellular growth, migration, and invasion, and repressed HCC cellular apoptosis. In vivo xenograft assays revealed that PAARH facilitated HCC tumor growth, repressed HCC cellular apoptosis, and promoted angiogenesis. These results supported that PAARH has oncogenic roles in HCC. Intriguingly, our findings revealed that PAARH not only modulated HCC cellular malignant phenotypes in vitro and in vivo, but also modulated angiogenesis in vitro and in vivo. The important roles of PAARH in HCC suggested that targeting PAARH represents a potential therapeutic strategy against HCC, which were also verified by loss-offunction experiments in this study. 
a

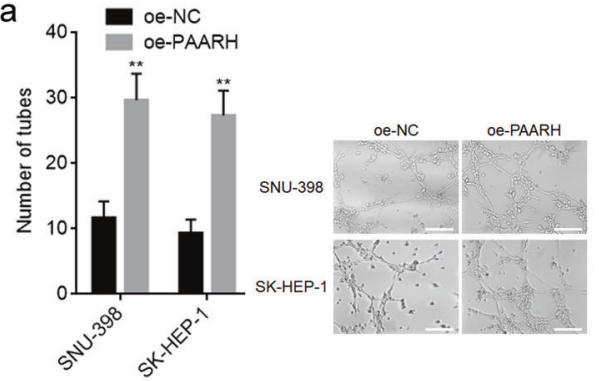

b

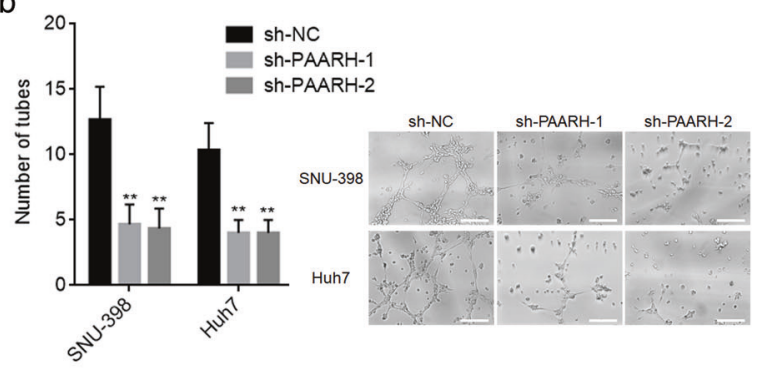

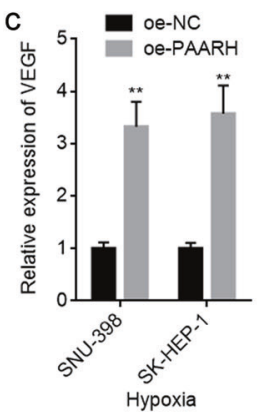

g

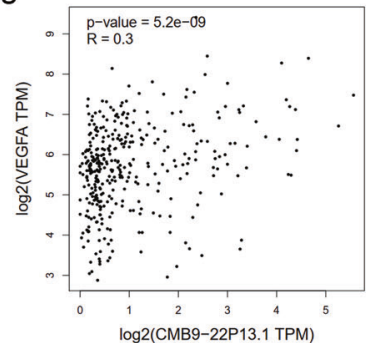

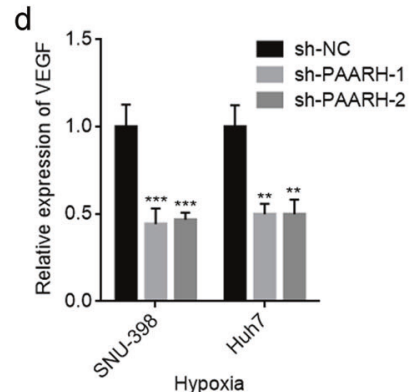

h

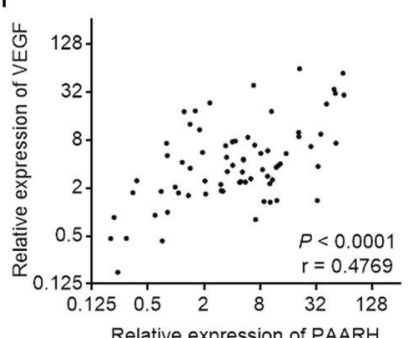

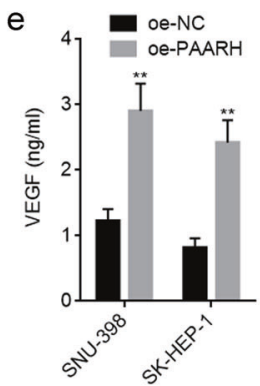

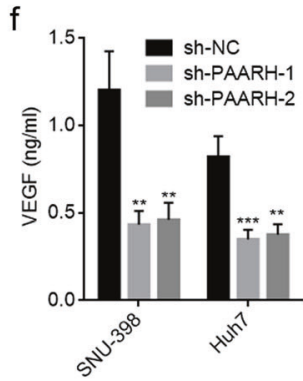

Fig. 7 PAARH promoted angiogenesis through upregulating VEGF. a The number of endothelial tubes formed by HUVEC treated with conditioned medium from SNU-398 and SK-HEP-1 cells with PAARH overexpression or control under hypoxia for $24 \mathrm{~h}$. Scale bars, $200 \mu \mathrm{m}$. b The number of endothelial tubes formed by HUVEC treated with conditioned medium from SNU-398 and Huh7 cells with PAARH silencing or control under hypoxia for $24 \mathrm{~h}$. Scale bars, $200 \mu \mathrm{m}$. c VEGF expression in SNU-398 and SK-HEP-1 cells with PAARH overexpression or control under hypoxia for $12 \mathrm{~h}$ was measured by qRT-PCR. d VEGF expression in SNU-398 and Huh7 cells with PAARH silencing or control under hypoxia for $12 \mathrm{~h}$ was measured by qRT-PCR. e ELISA measurement of VEGF protein level in conditioned medium from SNU-398 and SK-HEP-1 cells with PAARH overexpression or control under hypoxia for $24 \mathrm{~h}$. $\mathrm{f}$ ELISA measurement of VEGF protein level in conditioned medium from SNU-398 and Huh7 cells with PAARH silencing or control under hypoxia for $24 \mathrm{~h}$. $\mathbf{g}$ The correlation between VEGF and PAARH (CMB9-22P13.1) expression in HCC tissues according to TCGA LIHC dataset, analyzed by GEPIA. $\mathbf{h}$ The correlation between VEGF and PAARH expression in our HCC cohort. $n=72, p<0.0001, r=0.4769$ by Spearman correlation analysis. i PAARH expression levels in HCC tissues with high or low microvessel density (MVD), indicated by CD31 IHC staining. ${ }^{* * *} p<0.0001$ by Mann-Whitney test. For a-f, results are shown as mean \pm SD based on three independent experiments. ${ }^{* *} p<0.01,{ }^{* * *} p<0.001$ by Student's $t$ test $(\mathbf{a}, \mathbf{c}, \mathbf{e})$ or one-way ANOVA followed by Dunnett's multiple comparisons test $(\mathbf{b}, \mathbf{d}, \mathbf{f})$.

Mechanistic investigation revealed that PAARH exerted dual mechanisms (Fig. 8i). Cytoplasmic PAARH bound miR-6760-5p, miR-6512-3p, miR-1298-5p, miR-6720-5p, miR-4516, and miR-6782$5 p$, and relieved the repressive roles of these miRNAs on HOTTIP. Thus, PAARH functioned as a ceRNA to upregulate HOTTIP in HCC. HOTTIP is frequently reported as an oncogenic IncRNA in several cancers, including acute myeloid leukemia, head and neck squamous cell carcinoma, and HCC $[52,53,59]$. Here, functional rescue assays revealed that HOTTIP is a critical downstream mediator of the oncogenic roles of PAARH in HCC cellular malignant phenotype. The positive correlation between PAARH and HOTTIP expression in clinical HCC tissues supported the modulation of HOTTIP by PAARH.

Furthermore, nuclear PAARH was found to physically bind HIF$1 a$, recruited HIF-1a to VEGF promoter, and activated VEGF expression. The modulation of VEGF by PAARH is hypoxia dependent. HIF-1a protein is stabilized by hypoxia and translocated into nuclei to activate its targeted genes, such as VEGF [60]. Here, our findings identified HIF-1a as an interaction partner of PAARH. The binding of PAARH to HIF-1a did not change HIF-1a protein levels, but promoted the recruitment of HIF-1a to VEGF promoter, leading to the upregulation of VEGF expression. Thus, PAARH represents a novel regulator of hypoxia signaling in HCC. The positive expression between PAARH and VEGF expression, PAARH expression and microvascular density in HCC tissues supported the roles of PAARH in HCC angiogenesis. Previous reports have identified several factors regulating HIF-1a/VEGF signaling axis-induced angiogenesis in $\mathrm{HCC}$, such as $\mathrm{Cbx} 4$ and Hepatitis C virus glycoprotein [14, 19]. In this study, we showed that IncRNA is another class of important factor regulating HIF-1a/ VEGF-induced angiogenesis in HCC.

In conclusion, this study identified PAARH as a novel HCCrelated IncRNA, which was frequently upregulated in HCC and associated with BCLC stage, poor differentiation, microvascular 


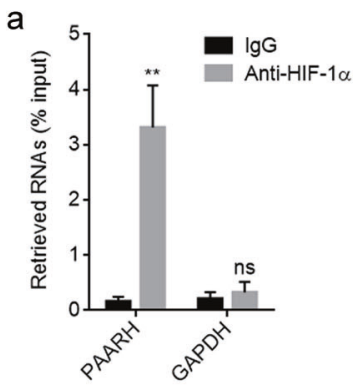

b
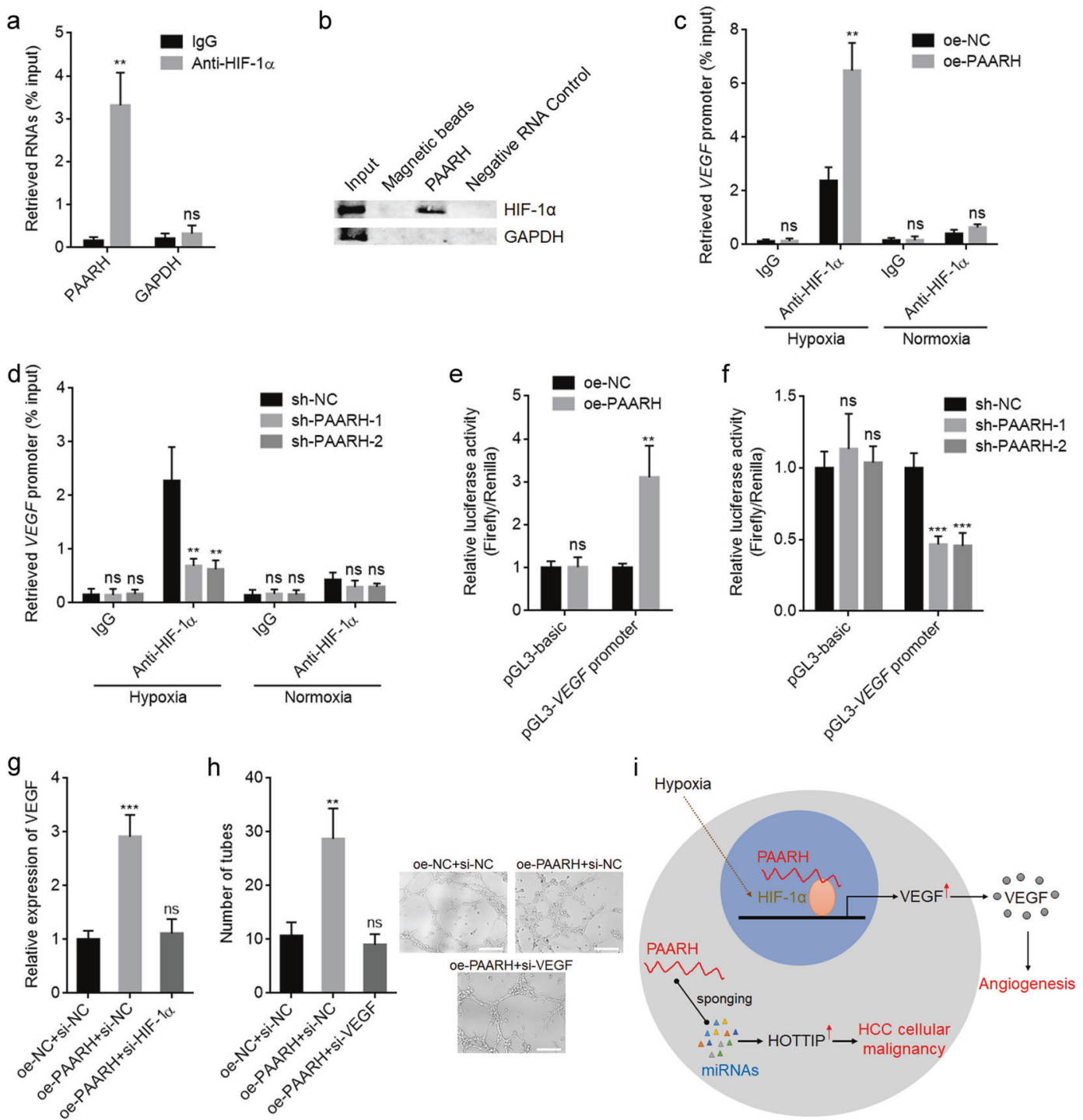

Fig. 8 PAARH physically bound to HIF-1a, recruited HIF-1a to VEGF promoter, and activated VEGF transcription. a RIP assays followed by qRT-PCR to detect RNAs bound to HIF-1 $\alpha$. b RNA pull-down experiments using RNA end-labeled with desthiobiotin, followed by western blot to detect proteins bound to PAARH. c SNU-398 cells with PAARH overexpression or control were incubated under hypoxia or normoxia for $24 \mathrm{~h}$, followed by ChIP with anti-HIF-1 $\alpha$ antibody or nonrelated IgG. Precipitated DNAs were measured by qRT-PCR for VEGF promoter. d SNU398 cells with PAARH silencing or control were incubated under hypoxia or normoxia for $24 \mathrm{~h}$, followed by ChIP with anti-HIF-1 $\alpha$ antibody or nonrelated IgG. Precipitated DNAs were measured by qRT-PCR for VEGF promoter. e Luciferase activity in SNU-398 cells with PAARH overexpression or control after transfection of luciferase reporters containing VEGF promoter or nothing. Results are shown as the relative ratio of firefly luciferase activity to renilla luciferase activity. $\mathbf{f}$ Luciferase activity in SNU-398 cells with PAARH silencing or control after transfection of luciferase reporters containing VEGF promoter or nothing. Results are shown as the relative ratio of firefly luciferase activity to renilla luciferase activity. g VEGF expression in SNU-398 cells with PAARH overexpression and concurrent HIF-1 $\alpha$ depletion under hypoxia for $12 \mathrm{~h}$ was measured by qRT-PCR. $\mathbf{h}$ The number of endothelial tubes formed by HUVEC treated with conditioned medium from SNU-398 cells with PAARH overexpression and concurrent VEGF depletion under hypoxia for $24 \mathrm{~h}$. Scale bars, $200 \mu \mathrm{m}$. i Schematic model of the roles of PAARH in regulating HCC cellular malignancy and angiogenesis. Results are shown as mean \pm SD based on three independent experiments. ${ }^{* *} p<0.01,{ }^{* *} p<0.001$, ns not significant, by Student's $t$ test $(\mathbf{a}, \mathbf{c}, \mathbf{e})$ or one-way ANOVA followed by Dunnett's multiple comparisons test (d, $\left.\mathbf{f}-\mathbf{h}\right)$.

invasion, poor overall survival, and poor disease-free survival. PAARH enhanced HCC cellular malignancies via sponging miRNAs and further upregulating HOTTIP. PAARH also promoted angiogenesis via binding HIF-1a and further increasing VEGF expression (Fig. 8i). These data implied PAARH as a potential prognostic biomarker and therapeutic target for HCC.

\section{DATA AVAILABILITY}

The datasets generated and/or analyzed during the current study are available from the corresponding author on reasonable request.

\section{REFERENCES}

1. Sung H, Ferlay J, Siegel RL, Laversanne M, Soerjomataram I, Jemal A, et al. Global Cancer Statistics 2020: GLOBOCAN Estimates of Incidence and Mortality Worldwide for 36 Cancers in 185 Countries. CA Cancer J Clin. 2021;71:209-49.

2. Villanueva A. Hepatocellular Carcinoma. N Engl J Med. 2019;380:1450-62.

3. Fountzilas C, Gupta M, Lee S, Krishnamurthi S, Estfan B, Wang K, et al. A multicentre phase $1 \mathrm{~b} / 2$ study of tivozanib in patients with advanced inoperable hepatocellular carcinoma. Br J Cancer. 2020;122:963-70.

4. Briggs A, Daniele B, Dick K, Evans TRJ, Galle PR, Hubner RA, et al. Covariateadjusted analysis of the Phase 3 REFLECT study of lenvatinib versus sorafenib in the treatment of unresectable hepatocellular carcinoma. $\mathrm{Br} J$ Cancer. 2020;122:1754-9. 
5. Liu WR, Tian MX, Tang Z, Fang Y, Zhou YF, Song SS, et al. Nine-factor-based immunohistochemistry classifier predicts recurrence for early-stage hepatocellular carcinoma after curative resection. Br J Cancer. 2020;123:92-100.

6. Dolgormaa G, Harimoto N, Ishii N, Yamanaka T, Hagiwara K, Tsukagoshi M, et al. Mac-2-binding protein glycan isomer enhances the aggressiveness of hepatocellular carcinoma by activating mTOR signaling. Br J Cancer. 2020;123:1145-53.

7. Tsui YM, Chan LK, Ng IO. Cancer stemness in hepatocellular carcinoma: mechanisms and translational potential. Br J Cancer. 2020;122:1428-40.

8. Kairaluoma V, Kemi N, Pohjanen VM, Saarnio J, Helminen O. Tumour budding and tumour-stroma ratio in hepatocellular carcinoma. Br J Cancer. 2020;123:38-45.

9. Wu J, Guo L, Qiu X, Ren Y, Li F, Cui W, et al. Genkwadaphnin inhibits growth and invasion in hepatocellular carcinoma by blocking DHCR24-mediated cholesterol biosynthesis and lipid rafts formation. Br J Cancer. 2020;123:1673-85.

10. Craig SG, Humphries MP, Alderdice M, Bingham V, Richman SD, Loughrey MB, et al. Immune status is prognostic for poor survival in colorectal cancer patients and is associated with tumour hypoxia. Br J Cancer. 2020;123:1280-8.

11. Bernauer C, Man YKS, Chisholm JC, Lepicard EY, Robinson SP, Shipley JM. Hypoxia and its therapeutic possibilities in paediatric cancers. $\mathrm{Br} J$ Cancer. 2021;124:539-51.

12. Sivalingam VN, Latif A, Kitson S, McVey R, Finegan KG, Marshall K, et al. Hypoxia and hyperglycaemia determine why some endometrial tumours fail to respond to metformin. Br J Cancer. 2020;122:62-71.

13. Hou J, Zhang H, Liu J, Zhao Z, Wang J, Lu Z, et al. YTHDF2 reduction fuels inflammation and vascular abnormalization in hepatocellular carcinoma. Mol Cancer. 2019;18:163.

14. Li J, Xu Y, Long XD, Wang W, Jiao HK, Mei Z, et al. Cbx4 governs HIF-1alpha to potentiate angiogenesis of hepatocellular carcinoma by its SUMO E3 ligase activity. Cancer Cell. 2014;25:118-31.

15. Hou $P$, Shi $P$, Jiang $T$, Yin $H$, Chu S, Shi M, et al. DKC1 enhances angiogenesis by promoting HIF-1alpha transcription and facilitates metastasis in colorectal cancer. Br J Cancer. 2020;122:668-79.

16. Yoon NA, Jung SJ, Choi SH, Ryu JH, Mani M, Lee UH, et al. DRG2 supports the growth of primary tumors and metastases of melanoma by enhancing VEGF-A expression. FEBS J. 2020;287:2070-86.

17. Song M, Pan Q, Yang J, He J, Zeng J, Cheng S, et al. Galectin-3 favours tumour metastasis via the activation of beta-catenin signalling in hepatocellular carcinoma. Br J Cancer. 2020;123:1521-34.

18. Cheng H, Cao X, Min X, Zhang X, Kong Q, Mao Q, et al. Heat-Shock protein A12A is a novel PCNA-binding protein and promotes hepatocellular carcinoma growth. FEBS J. 2020;287:5464-77.

19. Wilson GK, Brimacombe CL, Rowe IA, Reynolds GM, Fletcher NF, Stamataki Z, et al. A dual role for hypoxia inducible factor-1alpha in the hepatitis $C$ virus lifecycle and hepatoma migration. J Hepatol. 2012;56:803-9.

20. Lorenzi L, Chiu HS, Avila Cobos F, Gross S, Volders PJ, Cannoodt R, et al. The RNA Atlas expands the catalog of human non-coding RNAs. Nat Biotechnol. 2021:39:1453-65.

21. Hanniford D, Ulloa-Morales A, Karz A, Berzoti-Coelho MG, Moubarak RS, SanchezSendra B, et al. Epigenetic Silencing of CDR1as Drives IGF2BP3-Mediated Melanoma Invasion and Metastasis. Cancer Cell. 2020;37:55-70 e15.

22. Gao N, Hu J, He B, Ji Z, Hu X, Huang J, et al. Endogenous promoter-driven sgRNA for monitoring the expression of low-abundance transcripts and IncRNAs. Nat Cell Biol. 2021;23:99-108.

23. Liu SJ, Dang HX, Lim DA, Feng FY, Maher CA. Long noncoding RNAs in cancer metastasis. Nat Rev Cancer. 2021;21:446-60.

24. Sur S, Ray, RB. Emerging role of IncRNA ELDR in development and cancer. FEBS J. 2021. https://doi.org/10.1111/febs.15876 [Ahead of print].

25. Ono K, Horie T, Baba O, Kimura M, Tsuji S, Rodriguez RR, et al. Functional noncoding RNAs in vascular diseases. FEBS J. 2021;288:6315-30.

26. Esposito R, Bosch N, Lanzos A, Polidori T, Pulido-Quetglas C, Johnson R. Hacking the cancer genome: profiling therapeutically actionable long non-coding RNAs using CRISPR-Cas9 screening. Cancer Cell. 2019;35:545-57.

27. Li G, Kryczek I, Nam J, Li X, Li S, Li J, et al. LIMIT is an immunogenic IncRNA in cancer immunity and immunotherapy. Nat Cell Biol. 2021;23:526-37.

28. Zhang Y, Li Y, Hu Q, Xi Y, Xing Z, Zhang Z, et al. The IncRNA H19 alleviates muscular dystrophy by stabilizing dystrophin. Nat Cell Biol. 2020;22:1332-45.

29. Daneshvar K, Ardehali MB, Klein IA, Hsieh FK, Kratkiewicz AJ, Mahpour A, et al. InCRNA DIGIT and BRD3 protein form phase-separated condensates to regulate endoderm differentiation. Nat Cell Biol. 2020;22:1211-22.

30. Mo S, Zhang L, Dai W, Han L, Wang R, Xiang W, et al. Antisense IncRNA LDLRAD4AS1 promotes metastasis by decreasing the expression of LDLRAD4 and predicts a poor prognosis in colorectal cancer. Cell Death Dis. 2020;11:155.

31. Li Y, Yan G, Zhang J, Chen W, Ding T, Yin Y, et al. LncRNA HOXA11-AS regulates calcium oxalate crystal-induced renal inflammation via miR-124-3p/MCP-1. J Cell Mol Med. 2020;24:238-49.
32. Maguire EM, Xiao Q. Noncoding RNAs in vascular smooth muscle cell function and neointimal hyperplasia. FEBS J. 2020;287:5260-83.

33. Yuan JH, Yang F, Wang F, Ma JZ, Guo YJ, Tao QF, et al. A long noncoding RNA activated by TGF-beta promotes the invasion-metastasis cascade in hepatocellular carcinoma. Cancer Cell. 2014;25:666-81.

34. Zhu XT, Yuan JH, Zhu TT, Li YY, Cheng XY. Long noncoding RNA glypican 3 (GPC3) antisense transcript 1 promotes hepatocellular carcinoma progression via epigenetically activating GPC3. FEBS J. 2016;283:3739-54.

35. Wen Z, Lian L, Ding H, Hu Y, Xiao Z, Xiong K, et al. LncRNA ANCR promotes hepatocellular carcinoma metastasis through upregulating HNRNPA1 expression. RNA Biol. 2020;17:381-94.

36. Li H, Yan R, Chen W, Ding X, Liu J, Chen G, et al. Long non coding RNA SLC26A4AS1 exerts antiangiogenic effects in human glioma by upregulating NPTX1 via NFKB1 transcriptional factor. FEBS J. 2021;288:212-28.

37. Shen W, Huang B, He Y, Shi L, Yang J. Long non-coding RNA RP11-820 promotes extracellular matrix production via regulating miR-3178/MYOD1 in human trabecular meshwork cells. FEBS J. 2020;287:978-90.

38. Liu HT, Ma RR, Lv BB, Zhang H, Shi DB, Guo XY, et al. LncRNA-HNF1A-AS1 functions as a competing endogenous RNA to activate PI3K/AKT signalling pathway by sponging miR-30b-3p in gastric cancer. Br J Cancer. 2020;122:1825-36.

39. Li MM, Liu XH, Zhao YC, Ma XY, Zhou YC, Zhao YX, et al. Long noncoding RNA KCNQ1OT1 promotes apoptosis in neuroblastoma cells by regulating miR-2965p/Bax axis. FEBS J. 2020;287:561-77.

40. Kim TW, Lee YS, Yun NH, Shin CH, Hong HK, Kim HH, et al. MicroRNA-17-5p regulates EMT by targeting vimentin in colorectal cancer. Br J Cancer. 2020;123:1123-30.

41. Liang $Y$, Zhu D, Hou L, Wang Y, Huang X, Zhou C, et al. MiR-107 confers chemoresistance to colorectal cancer by targeting calcium-binding protein $39 . \mathrm{Br} J$ Cancer. 2020;122:705-14.

42. Li JK, Chen C, Liu JY, Shi JZ, Liu SP, Liu B, et al. Long noncoding RNA MRCCAT1 promotes metastasis of clear cell renal cell carcinoma via inhibiting NPR3 and activating p38-MAPK signaling. Mol Cancer. 2017;16:111.

43. Acha-Sagredo A, Uko B, Pantazi P, Bediaga NG, Moschandrea C, Rainbow L, et al. Long non-coding RNA dysregulation is a frequent event in non-small cell lung carcinoma pathogenesis. Br J Cancer. 2020;122:1050-8.

44. Tang Z, Li C, Kang B, Gao G, Li C, Zhang Z. GEPIA: a web server for cancer and normal gene expression profiling and interactive analyses. Nucleic Acids Res. 2017:45:W98-102.

45. Wang L, Park HJ, Dasari S, Wang S, Kocher JP, Li W. CPAT: coding-potential assessment tool using an alignment-free logistic regression model. Nucleic Acids Res. 2013;41:e74

46. Kang YJ, Yang DC, Kong L, Hou M, Meng YQ, Wei L, et al. CPC2: a fast and accurate coding potential calculator based on sequence intrinsic features. Nucleic Acids Res. 2017;45:W12-16.

47. Paraskevopoulou MD, Vlachos IS, Karagkouni D, Georgakilas G, Kanellos I, Vergoulis $\mathrm{T}$, et al. DIANA-LncBase v2: indexing microRNA targets on non-coding transcripts. Nucleic Acids Res. 2016:44:D231-8.

48. Muppirala UK, Honavar VG, Dobbs D. Predicting RNA-protein interactions using only sequence information. BMC Bioinforma. 2011;12:489.

49. Yuan JH, Liu XN, Wang TT, Pan W, Tao QF, Zhou WP, et al. The MBNL3 splicing factor promotes hepatocellular carcinoma by increasing PXN expression through the alternative splicing of IncRNA-PXN-AS1. Nat Cell Biol. 2017;19:820-32.

50. Pu J, Wang J, Wei H, Lu T, Wu X, Wu Y, et al. IncRNA MAGI2-AS3 prevents the development of $\mathrm{HCC}$ via recruiting KDM1A and promoting $\mathrm{H} 3 \mathrm{~K} 4 \mathrm{me} 2$ demethylation of the RACGAP1 promoter. Mol Ther Nucleic Acids. 2019;18:351-62.

51. Zhao R, Zhang X, Zhang Y, Zhang Y, Yang Y, Sun Y, et al. HOTTIP predicts poor survival in gastric cancer patients and contributes to cisplatin resistance by sponging miR-216a-5p. Front Cell Dev Biol. 2020;8:348.

52. Luo H, Zhu G, Xu J, Lai Q, Yan B, Guo Y, et al. HOTTIP IncRNA promotes hematopoietic stem cell self-renewal leading to AML-like disease in mice. Cancer Cell. 2019;36:645-59 e648.

53. Quagliata L, Matter MS, Piscuoglio S, Arabi L, Ruiz C, Procino A, et al. Long noncoding RNA HOTTIP/HOXA13 expression is associated with disease progression and predicts outcome in hepatocellular carcinoma patients. Hepatology. 2014;59:911-23.

54. Lin D, Wu J. Hypoxia inducible factor in hepatocellular carcinoma: a therapeutic target. World J Gastroenterol. 2015;21:12171-8.

55. Ma L, Hernandez MO, Zhao $Y$, Mehta M, Tran B, Kelly $M$, et al. Tumor cell biodiversity drives microenvironmental reprogramming in liver cancer. Cancer Cell. 2019;36:418-30 e416.

56. Horikawa N, Abiko K, Matsumura N, Baba T, Hamanishi J, Yamaguchi K, et al. AntiVEGF therapy resistance in ovarian cancer is caused by GM-CSF-induced myeloidderived suppressor cell recruitment. Br J Cancer. 2020;122:778-88.

57. Liu K, Min XL, Peng J, Yang K, Yang L, Zhang XM. The Changes of HIF-1alpha and VEGF Expression After TACE in Patients With Hepatocellular Carcinoma. J Clin Med Res. 2016;8:297-302. 
58. Hajiev S, Allara E, Motedayen Aval L, Arizumi T, Bettinger D, Pirisi M, et al. Impact of age on sorafenib outcomes in hepatocellular carcinoma: an international cohort study. Br J Cancer. 2021;124:407-13.

59. Sun Q, Zhang SY, Zhao JF, Han XG, Wang HB, Sun ML. HIF-1alpha or HOTTIP/CTCF promotes head and neck squamous cell carcinoma progression and drug resistance by targeting HOXA9. Mol Ther Nucleic Acids. 2020;20:164-75.

60. Zhang J, Zhang Y, Mo F, Patel G, Butterworth K, Shao C, et al. The roles of HIF1alpha in radiosensitivity and radiation-induced bystander effects under hypoxia. Front Cell Dev Biol. 2021;9:637454.

\section{ACKNOWLEDGEMENTS}

This work was supported by the Guangxi Natural Science Foundation Project (2019GXNSFBA245023, 2020GXNSFAA259019) and Baise Scientific Research and Technology Development Programme (BKZ2020-47-23).

\section{AUTHOR CONTRIBUTIONS}

JP and HW designed the study. HW, ZX, LC, QW, ZH, GL, and WL performed the experiments. JP, HW, QT, and JW analyzed the data. JP and HW are the major contributors in writing the manuscript. All authors read and approved the final manuscript.

\section{COMPETING INTERESTS}

The authors declare no competing interests.

\section{ADDITIONAL INFORMATION}

Supplementary information The online version contains supplementary material available at https://doi.org/10.1038/s41419-022-04505-5.

Correspondence and requests for materials should be addressed to Jian Pu.

Reprints and permission information is available at http://www.nature.com/ reprints

Publisher's note Springer Nature remains neutral with regard to jurisdictional claims in published maps and institutional affiliations.

Attribution 4.0 International License, which permits use, sharing, adaptation, distribution and reproduction in any medium or format, as long as you give appropriate credit to the original author(s) and the source, provide a link to the Creative Commons license, and indicate if changes were made. The images or other third party material in this article are included in the article's Creative Commons license, unless indicated otherwise in a credit line to the material. If material is not included in the article's Creative Commons license and your intended use is not permitted by statutory regulation or exceeds the permitted use, you will need to obtain permission directly from the copyright holder. To view a copy of this license, visit http://creativecommons. org/licenses/by/4.0/.

(c) The Author(s) 2022 